\title{
Simulation-based Optimisation of Maintenance Systems: Industrial Case Studies
}

\author{
Abdullah Alrabghi*, Ashutosh Tiwari** and Mark Savill ${ }^{* * *}$ \\ * Department of Industrial Engineering, University of Jeddah, Jeddah, Saudi Arabia \\ ** Manufacturing Department, Cranfield University, Cranfield, United Kingdom \\ *** Propulsion Engineering Centre, Cranfield University, Cranfield, United Kingdom \\ E-Mail: aalrabghi@uj.edu.sa, a.tiwari@cranfield.ac.uk, mark.savill@cranfield.ac.uk
}

\begin{abstract}
Investigating the optimum blend of maintenance strategies for a given manufacturing system is a continuing concern amongst maintenance academics and professionals. Recent evidence suggests that little research is conducted on the simulation optimisation of maintenance in industrial systems. This study was designed to make an important contribution to the field of simulation-based optimisation of maintenance by presenting two empirical case studies: a tyre re-treading factory and a petro-chemical plant. It is one of the first to optimise various maintenance strategies simultaneously with their parameters in industrial manufacturing systems while considering production dynamics. Stochastic Discrete Event Simulation models were developed and connected to a Multi-Objective Optimisation engine. Various maintenance strategies were investigated including Corrective Maintenance, Preventive Maintenance, Opportunistic Maintenance and Condition-Based Maintenance. The results of this research suggest that over-looking the optimisation of maintenance on the strategic level may lead to sub-optimal solutions. In addition, it appears that traditional trade-offs between maintenance cost and production throughput are not present in some maintenance systems. This is an interesting observation that requires further investigation and experimentation.
\end{abstract}

Keywords: simulation, maintenance, Discrete Event Simulation, Optimisation, industrial case studies

\section{List of Abbreviations:}

$\begin{array}{ll}\text { CBM } & \text { Condition Based Maintenance } \\ \text { CM } & \text { Corrective Maintenance } \\ \text { DES } & \text { Discrete Event Simulation } \\ \text { GA } & \text { Genetic Algorithms } \\ \text { LCU } & \text { Local Currency Unit } \\ \text { MOO } & \text { Multi-Objective Optimisation } \\ \text { MTBF } & \text { Mean Time Between Failures } \\ \text { NSGA II } & \text { Non-dominated Sorting Genetic Algorithms II } \\ \text { OM } & \text { Opportunistic Maintenance } \\ \text { PK } & \text { zero-to-peak } \\ \text { PM } & \text { Preventive Maintenance } \\ \text { SSP } & \text { Solid State Polycondensation } \\ \text { TTF } & \text { Time To Failure }\end{array}$




\section{List of Notations:}

$\begin{array}{ll}i & \text { A single asset in the system where } i=1 \ldots n \\ \text { Labour } & \text { Number of maintenance technicians } \\ M A & \text { A single maintenance action resulting from a maintenance strategy } \\ M c_{i} & \text { Machine } i \\ M S_{i} & \text { Maintenance strategy for machine } i \\ n & \text { Total number of assets in the system } \\ P M f r e q_{i} & \text { Preventive maintenance frequency for machine } i \\ Q_{i} & \text { Order quantity for } S P_{i} \\ S_{i} & \text { Reorder level for } S P_{i} \\ S M A & \text { A scheduled maintenance action resulting from a maintenance strategy } \\ S P_{i} & \text { Spare part for machine } i \\ T & \text { simulation run length }\end{array}$

\section{Introduction}

Investigating the optimum blend of maintenance strategies for a given manufacturing system is a continuing concern amongst maintenance academics and professionals [1]. Increased throughput, higher asset availability and cost savings are some of the benefits gained from maintenance optimisation.

Industrial systems are becoming more complex making simulation the preferred modelling choice [2-4]. The inherited uncertainty in assets behaviour is one of the main contributors to the complexity of maintenance problems. This is further increased by variations in factors such as operating conditions, production schedules, spare parts policies and dependencies between components which affect the degradation pattern or the main maintenance performance measures. Increasing the number of assets in the system or the number of applicable maintenance strategies and policies will increase the number of decision variables leading to more complexity in the maintenance optimisation problem.

Recent evidence suggests that little research is conducted on the simulation optimisation of industrial case studies $[1 ; 5]$. This prospective study was designed to make an important contribution to the field of simulation-based optimisation of maintenance by presenting two empirical case studies. The research focus is on maintenance practices rather than particular simulation or optimization tools. Data is collected from a tyre re-treading factory (industrial case A) and a petro-chemical plant (industrial case B). As shown in Table 1, the two case studies were carefully selected to ensure that together they are able to validate the research results for key features in maintenance optimisation problems. The maintenance systems in the case studies vary in terms of sector, size, number of manufacturing processes and level of maintenance documentation. when considered together, the case studies ensure the generality of the research results. 
Table 1 Main features of industrial cases

\begin{tabular}{|l|l|l|}
\hline & Industrial case A & Industrial case B \\
\hline Sector & Tyre re-treading & Petro-chemicals \\
\hline Company size & Small <50 employees & Large $>300$ employees \\
\hline $\begin{array}{l}\text { Number of } \\
\text { manufacturing } \\
\text { processes }\end{array}$ & 11 & 4 \\
\hline $\begin{array}{l}\text { Maintenance } \\
\text { documentation }\end{array}$ & Minimal & Updated regularly in SAP \\
\hline $\begin{array}{l}\text { Applicable maintenance } \\
\text { strategies }\end{array}$ & CM and PM & CM, OM and CBM \\
\hline Optimisation scope & Maintenance & Maintenance \\
\hline $\begin{array}{l}\text { Optimisation objectives } \\
\text { Max throughput } \\
\text { Min maintenance cost }\end{array}$ & $\begin{array}{l}\text { Max throughput } \\
\text { Min maintenance cost }\end{array}$ \\
\hline Decision variables & $\begin{array}{l}\text { Maintenance strategy } \\
\text { PM frequency } \\
\text { Maintenance technicians }\end{array}$ & $\begin{array}{l}\text { Maintenance strategy } \\
\text { CBM inspection frequency } \\
\text { CBM threshold }\end{array}$ \\
\hline
\end{tabular}

This paper first outlines the methodology including data collection and analysis, the approach to modelling maintenance systems and optimisation algorithms utilised in the study. Sections 3 and 4 present the findings of the industrial case studies. Cross case examination and analysis are conducted in section 5 and conclusions are presented in section 6.

\section{Methodology}

\subsection{Data Collection}

During the initial visits, discussions were conducted to determine which production line will be the focus of the research. That is usually decided based on the most critical assets from the maintenance point of view where maintenance managers are faced with continuous challenges in keeping the equipment available as planned. Interviews and site visits were then scheduled with the relevant production manager to understand the manufacturing process in detail. For data confidentiality purposes, the case studies will be labelled as industrial case A and industrial case B.

The data was collected mainly from manuals and records. This was further clarified by engineers and managers in the industry. However, if the required data was not available due to poor documentation or confidentiality, approximate distributions such as Uniform or Triangular distributions are utilised by collecting available data such as maximum, mode and minimum values [6].

Collected data included a list of all equipment in the production line, the detailed record for all maintenance interventions including durations, spare parts involved, cost estimations, maintenance technicians as well as PM plans and execution.

\subsection{Data Analysis}

Raw data needed to be analysed in order to use it as an input to the simulation optimisation process. For example, raw data included the start and finish date 
and time of each maintenance intervention for all assets. Therefore, the durations had to be calculated and separated for each asset. In addition, data for different maintenance strategies had to be categorised and analysed independently.

In order to capture the variability in maintenance systems, stochastic data were fitted into statistical distributions [6]. The analysis included plotting the empirical data in a histogram. A statistical software package (Stat-Fit) was used to auto-fit the empirical data into theoretical distributions. At this stage, transforming some of the input data was required in order to obtain a better fit to theoretical distributions. The suggested distribution was further confirmed via goodness of fit tests as well as various graphical approaches such as Probability - Probability Plot and Quantile - Quantile Plot.

Witness - the simulation software- does not allow imposing minimum and maximum values on some statistical distributions, which presents a risk of producing infeasible high values in the simulation model [6]. Therefore, times for maintenance actions were restricted to the minimum and maximum values found in the empirical data.

If CBM is investigated in the maintenance system, the degradation process of assets needs to be modelled. Condition of assets is monitored by measuring the vibration levels. The convention used is to measure the vibration signal zero-to-peak (PK) regularly in $\mathrm{mm} / \mathrm{Sec}$. It is assumed that only maintenance interventions can enhance the state of assets and that the degradation process is stochastic with independent increments. Therefore, only ascending and stationary trends from the condition monitoring data were analysed. To enable the modelling of degradation increments, the increments are calculated over five day periods. The data points with no increments were considered as 'no changes in the condition indicator'. Minimum, mode and maximum data points are used as an input to a Triangular distribution that defines the degradation of the asset.

\subsection{Simulation Modelling}

Maintenance strategies were modelled according to the approach described by the authors [7]. It enables the modelling of non-identical multi-unit manufacturing systems without restrictions on maintenance or manufacturing characteristics (Figure 1). The approach can be integrated with DES manufacturing and spare parts models making it possible to build on the success DES achieved in these fields. Additional advantages of using DES include rapid modelling and visual interactive simulation. 


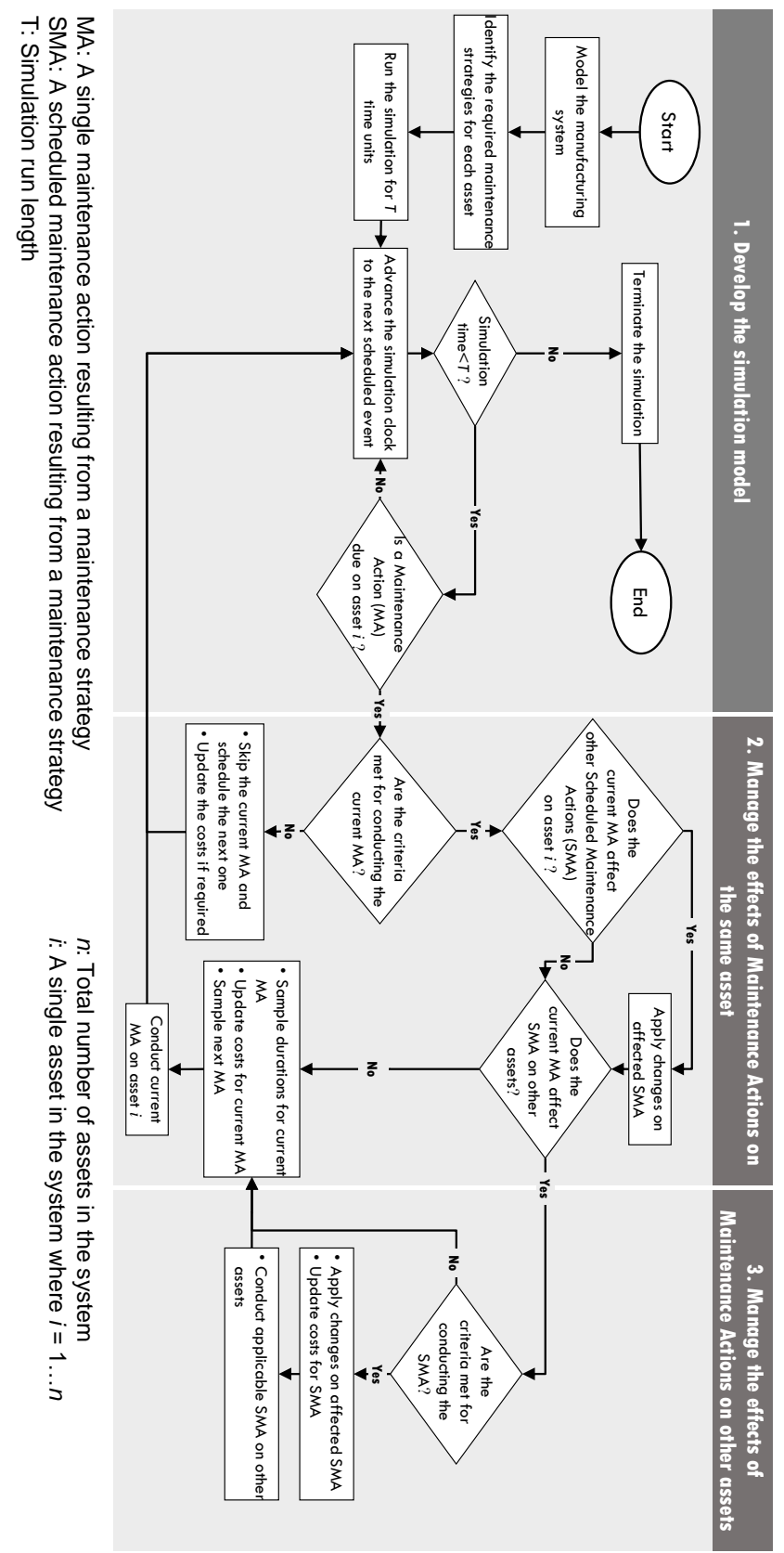

Figure 1 A generic approach for modelling maintenance strategies. Source [7]

The proposed approach enables the modelling of the complexity found in real maintenance systems. In particular, the approach enables the modelling of the following:

- Multi-unit manufacturing systems, without restrictions on the number of units

- Non-identical units, without restrictions placed on the manufacturing or the maintenance characteristics of units

- Several maintenance strategies and policies simultaneously

- Maintenance integrated with inter-related systems such as production and spare parts management 
Main assumptions include perfect maintenance where assets become as good as new following maintenance interventions and perfect inspections that reveal the real condition of the asset. As shown in Figure 2, MTBF is defined as the mean time between the start of any two consecutive failures.

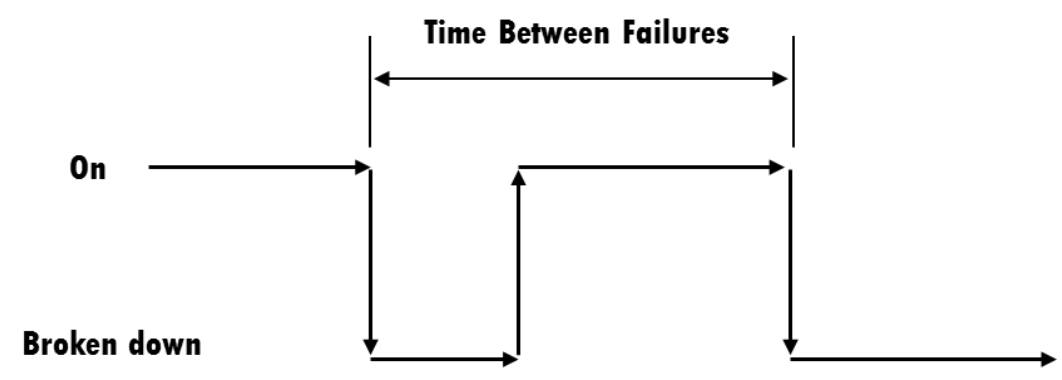

Figure 2 MTBF notation

Simulation models were developed by Witness 14 as it is already available within the research group. Each simulation is run for a number of replications to account for the variability arising from stochastic maintenance and production processes. Replications are conducted by running the same simulation model while changing the streams of random numbers used to sample from statistical distributions. A graphical method [6] is adopted to define a sufficient number of replications. It involves plotting the cumulative mean of the simulation output over a number of replications. The line becomes flat gradually which suggests that sufficient replications have been reached.

As simulation models start with empty conditions (no parts are present in the system), there is a chance of an initialisation bias. Introducing a warm up period enables the model to reach a steady state before beginning the optimisation process. Welch's method [8] cited in [6] is based on calculating the moving average of simulation output using the following formula:

$\bar{Y}_{i}(w)=\left\{\begin{array}{lr}\frac{\sum_{s=-(i-1)}^{i-1} \bar{Y}_{i+s}}{2 i-1} & \text { if } i=1, \ldots, w \\ \frac{\sum_{s=-w}^{w} \bar{Y}_{i+s}}{2 w+1} & \text { if } i=w+1, \ldots, m-w\end{array}\right.$

Where:

$\bar{Y}_{i}(w)=$ moving average of window size $w$

$\bar{Y}_{i}=$ time-series of output data (mean of the replications)

$i=$ period number

$m=$ number of periods in the simulation run

The moving average $\bar{Y}_{i}(w)$ is plotted in a line graph. The warm-up period is identified as the point in simulation time where the line becomes flat.

\subsection{Model Validation}

The simulation model was validated considering both white-box and black-box validation approaches [6]. In white-box validation, it is determined whether the internal construct of the model represent the real world with sufficient accuracy. 
Black-box validation however, aims to determine whether the overall model produce results with sufficient accuracy. The purpose of the simulation model is to represent the production line and its maintenance operations.

White-box validation methods were performed by the researcher and a simulation expert. It included the following:

- Checking the model code: continuously revising the code and checking the data and model logic.

- Visual checks: animating the simulation and monitoring the behaviour of various elements, running the simulation model event by event and comparing the expected behaviour of items against the model.

- Inspecting output reports for individual elements: This includes built-in reports such as asset utilisation, down times, repair times and average time a part spends in the system. In addition, specific output to trace asset degradation and the effect of maintenance actions were coded to be printed continuously for checks.

Likewise, black-box validation methods were adopted by comparing the simulation results to the current industrial systems. This includes production throughput, asset downtimes and asset availability. Historical data were used for the purpose of comparison. Additionally, knowledgeable experts from the concerned company were engaged to ensure valid representation of the model output.

\subsection{Simulation-based Optimisation}

In optimising the industrial maintenance systems, we follow the simulationbased optimisation framework developed by the authors [7]. It provides detailed assistance for optimising maintenance simulation models. The systematic methodology considers current issues relating to maintenance systems both in research and in practice such as uncertainty, complexity and multi-objective optimisation.

As illustrated in Figure 3, the framework is a step-by-step flow chart that guides a user in defining the optimisation scope, identifying applicable maintenance strategies, formulating the optimisation problem, selecting the optimisation algorithm, setting the simulation parameters and interpreting the results enabling practitioners and researchers to customise the maintenance problem to their specific needs. The framework provides detailed steps in two additional levels. 


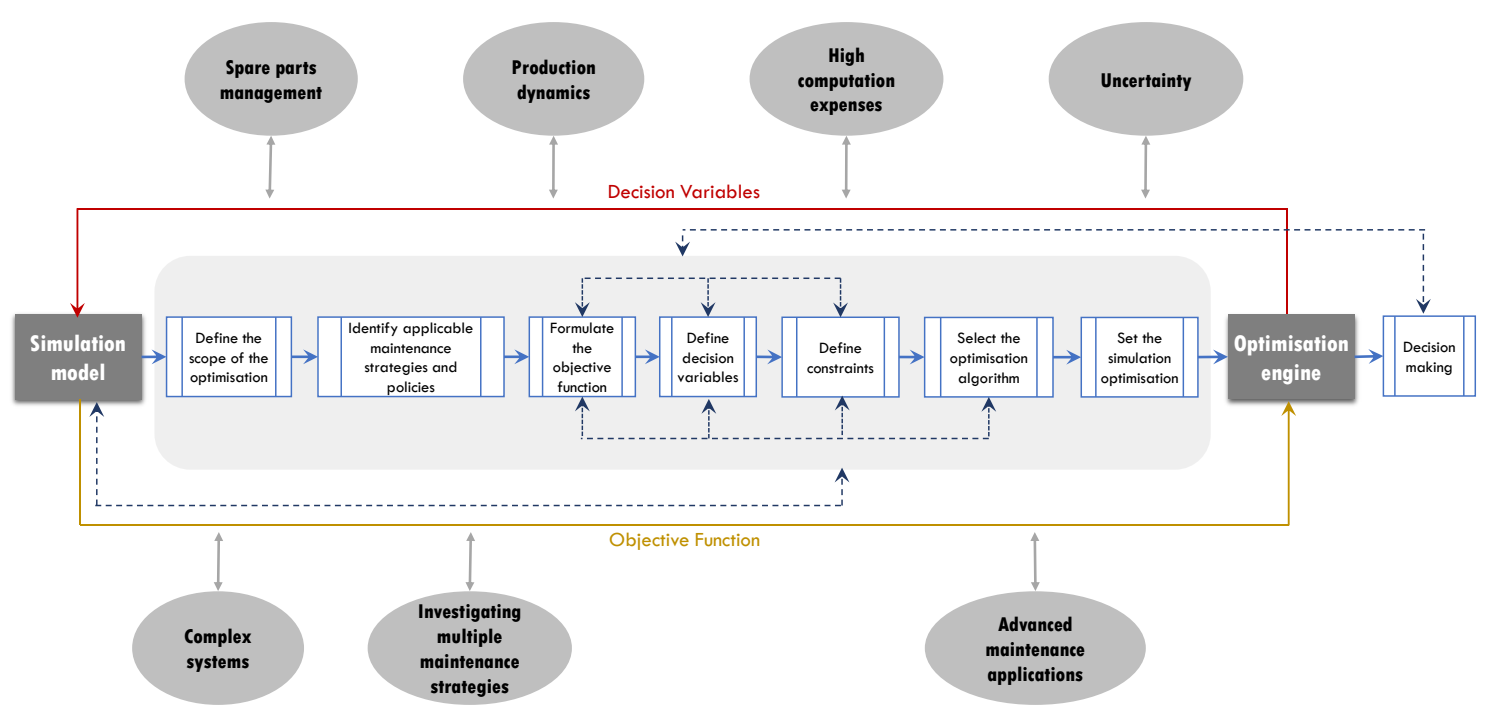

Figure 3 Simulation-based optimisation framework for maintenance systems on a high level. Source [13]

\subsection{Multi-Objective Optimisation}

The simulation model was linked to an optimisation engine to conduct MultiObjective Optimisation (MOO). An interface was developed to connect Witness to GAnetXL [9], a Genetic Algorithm Optimisation add-in for Microsoft Excel. The application is written in $\mathrm{C}++$ to allow interactions with Microsoft Excel.

GAnetXL employs GA, which is a population based evolutionary algorithm. The first population which contains the first set of decision variables is created randomly. The decision variables are sent to the simulation model for the purpose of evaluation. In order to produce the second set of solutions, a number of operators are applied including selection, crossover, mutation and elitism operators. The selection operator aims to choose from the old population to fill a mating pool giving more probability to better solutions. Crossover and mutation operators aim to create variations amongst some of the selected solutions in the mating pool to produce a new population. The elitism operator ensures that better solutions are kept from both old and new populations. In the current research, the optimisation process is terminated when it reaches the maximum number of generations [10].

MOO can result in a set of non-dominated solutions. In other words, a set of trade-off solutions where none of them achieve better than the others in all the objectives. GAnetXL solves multi-objective optimisation using Non-dominated Sorting Genetic Algorithms (NSGA II) where the elitism operator ensures the new populations incorporate the non-dominated solutions [10]. The crossover rate used in this research is 0.8 whereas the mutation rate is 0.05 . Similar values for these operators were used in simulation based optimisation of maintenance using GA [11; 12].

The simulation optimisation was run using population size of 50 for 100 generations. The number of generations is increased gradually if the algorithm is showing progress. Similarly, population sizes of 75 and 100 are used. Each 
combination of population size and generations was run using three different random seeds. Non-dominated solutions from the different random seeds were used to plot the data.

\section{Industrial Case A}

\subsection{Description of Factory A}

Industrial case A takes place in a tyre re-treading factory comprised of two main production lines:

- Trucks and lorries

- Tractors and heavy equipment

The production line for trucks is considered more critical as it is experiencing greater demand. Therefore it was selected for the case study. As illustrated in Figure 4, the production line involves eleven processes as follows:

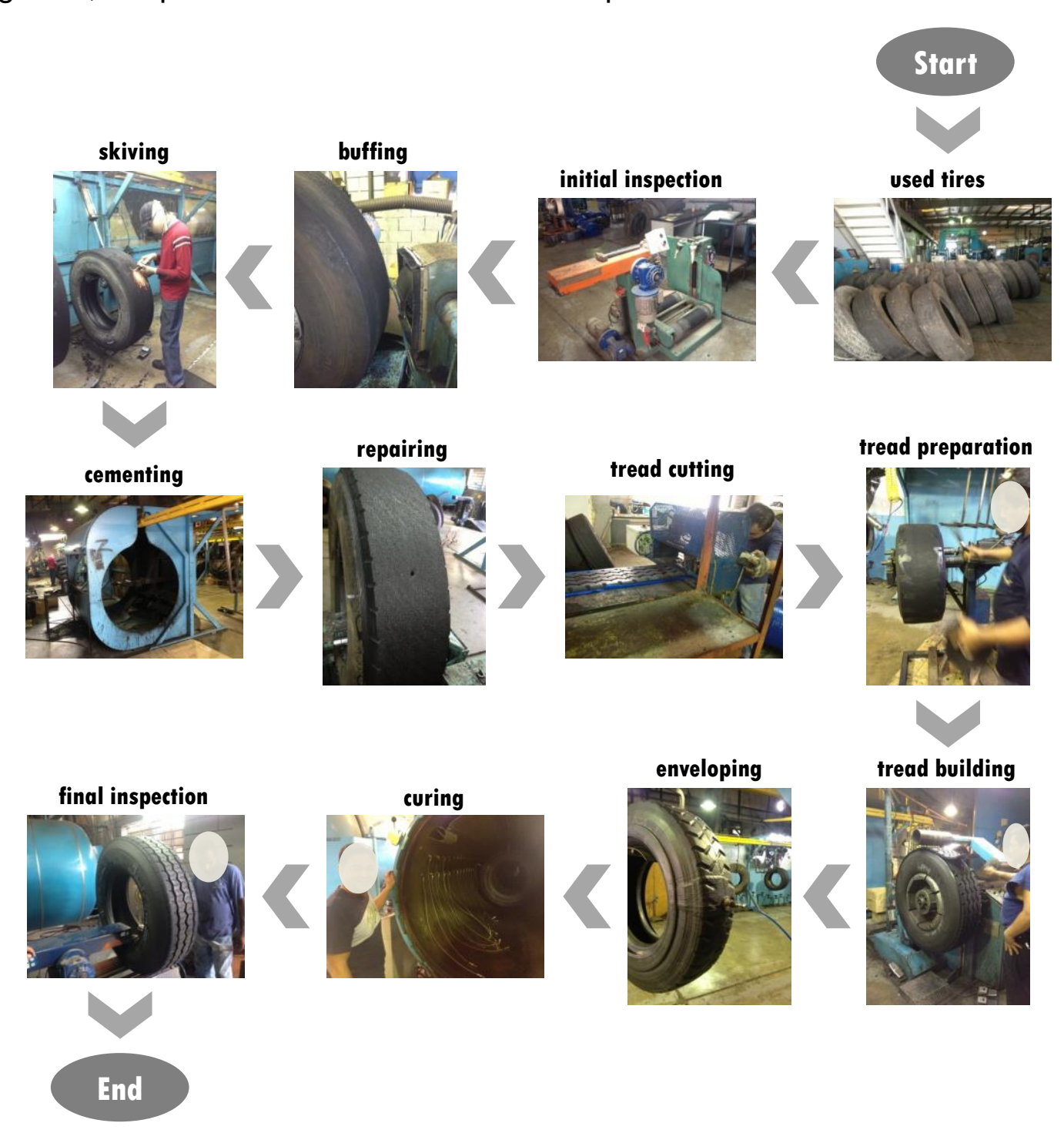

Figure 4 Tyre re-treading manufacturing process - trucks production line 
1. Initial inspection: casing is thoroughly inspected by a technician who determines if it is suitable for re-treading and if so the type of work to be performed on the tyre

2. Buffing: the worn tyre tread is entirely removed from the casing. The technician buffs and cuts the tyre to a specific radius and diameter on an expandable station

3. Skiving: embedded foreign objects and loss wires are removed to ensure a clean and solid surface

4. Cementing: a thin layer of concentrated rubber solution is sprayed on the casing

5. Repairing: minor defects such as small punctures and holes are fixed

6. Tread cutting: treads are prepared and cut for each tyre according to its size and customer requirements

7. Tread preparation and building: a new layer of compact pre-cured tread is built on the tyre casing. A thin layer of special bonding rubber is placed between the pre-cured tread and the casing

8. Enveloping: the tyre is bagged in a flexible envelope then vacuumed completely

9. Curing: the tyre is positioned in a heated chamber to start the process of vacuumisation under high pressure which results in a homogenous and permanent bonding of the pre-cured tread to the tyre casing

10. Unloading: taking the tyres from the chamber and separating it from the envelop

11. Final inspection: the re-treaded tyre is inspected thoroughly before shipping to customers

The cycle times for each process are shown in Table 2.

Table 2 Case A machine cycle times

\begin{tabular}{|l|l|c|l|l|}
\hline & Process & $\begin{array}{l}\text { Number of } \\
\text { workstations }\end{array}$ & Cycle time (hours) & $\begin{array}{l}\text { Setup time } \\
\text { (hours) }\end{array}$ \\
\hline 1 & Initial inspection & 1 & Triangle $(0.05,0.08,0.25)$ & N/A \\
\hline 2 & Buffing & 2 & Triangle $(0.08,0.13,0.25)$ & N/A \\
\hline 3 & Skiving & 3 & Triangle $(0.05,0.25,0.5)$ & N/A \\
\hline 4 & Cementing & 1 & Triangle $(0.08,0.1,0.12)$ & $\mathrm{N} / \mathrm{A}$ \\
\hline 5 & Repair & 2 & Triangle $(0,0.12,0.5)$ & $\mathrm{N} / \mathrm{A}$ \\
\hline 6 & Tread cutting & 1 & Triangle $(0.07,0.08,0.17)$ & $\mathrm{N} / \mathrm{A}$ \\
\hline 7 & $\begin{array}{l}\text { Tread preparation and } \\
\text { building }\end{array}$ & 1 & Triangle $(0.08,0.17,0.25)$ & $\mathrm{N} / \mathrm{A}$ \\
\hline 8 & Enveloping & 1 & Triangle $(0.08,0.12,0.20)$ & $\mathrm{N} / \mathrm{A}$ \\
\hline 9 & Curing & 1 & Triangle $(4,5,6)$ & 0.17 \\
\hline 10 & Unloading & 1 & Triangle $(0.003,0.03,0.08)$ & $\mathrm{N} / \mathrm{A}$ \\
\hline 11 & Final inspection & 1 & Triangle $(0.03,0.08,0.12)$ & $\mathrm{N} / \mathrm{A}$ \\
\hline
\end{tabular}

All machines require labour to operate except curing. However, the curing machine needs a labour to set it up which involves loading tyres to the chamber and switching the machine on. Therefore, the curing process can continue to work out of shift hours since it does not need any operators. As shown in Table 3 , most workers are multi-skilled which enables the production manager to reschedule the workforce regularly to ease bottlenecks. 
Table 3 Labour skills in case A

\begin{tabular}{|c|c|c|c|c|c|c|c|c|c|c|c|}
\hline & 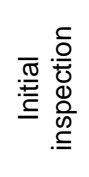 & 号 & 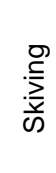 & 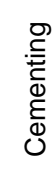 & $\begin{array}{l}\text { 产 } \\
\text { वें } \\
\widetilde{\alpha}\end{array}$ & 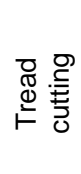 & 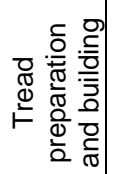 & 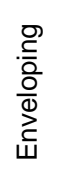 & 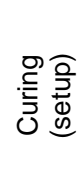 & 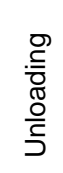 & 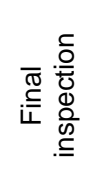 \\
\hline Labour 1 & $\checkmark$ & & $\checkmark$ & & & & $\checkmark$ & & & & \\
\hline Labour 2 & & $\checkmark$ & $\checkmark$ & & & $\checkmark$ & & & & & \\
\hline Labour 3 & & & $\checkmark$ & $\checkmark$ & & & & & & & \\
\hline Labour 4 & & & $\checkmark$ & $\checkmark$ & & & & & $\checkmark$ & $\checkmark$ & \\
\hline Labour 5 & & & & $\checkmark$ & & & & & $\checkmark$ & $\checkmark$ & \\
\hline Labour 6 & & & $\checkmark$ & $\checkmark$ & $\checkmark$ & & & & & & \\
\hline Labour 7 & & & & $\checkmark$ & $\checkmark$ & & & & & & \\
\hline Labour 8 & & & & & & $\checkmark$ & & & & & \\
\hline Labour 9 & & $\checkmark$ & & & & & $\checkmark$ & & & & \\
\hline Labour 10 & & & & & & & & & $\checkmark$ & $\checkmark$ & \\
\hline Labour 11 & & & & $\checkmark$ & & & & & & & $\checkmark$ \\
\hline
\end{tabular}

There are two possible rejection scenarios for tyres within the production line:

1. $30 \%$ are rejected at the initial inspection mainly because they are deemed unsuitable for re-treading

2. $5 \%$ are rejected from the skiving area. The operator can see defects in the case now more clearly having the old tread removed. This results in finding some tyres that are not suitable for re-treading

In addition, there is a rework loop:

- $5 \%$ of tyres fail the final inspection stage and have to go back to tread preparation and building process and then proceed again as normal

Figure 5 shows the simulation layout for Case A.

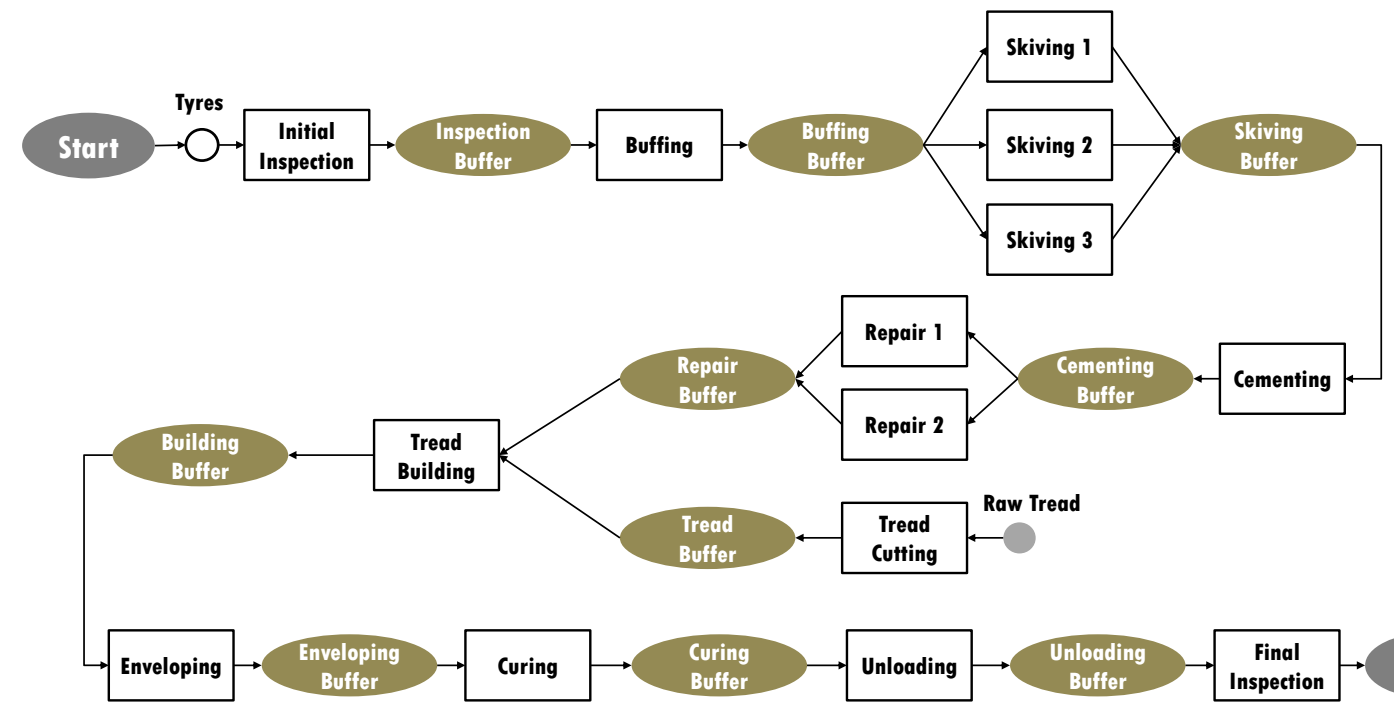

Figure 5 Case A simulation layout 


\subsection{Maintenance Operations}

Documentation of maintenance interventions is minimal. No records are held for downtimes and reasons of failure. In addition, $\mathrm{CM}$ is the only applied maintenance strategy. The explanation given by the factory management was that most breakdowns can be fixed manually by the operator in a relatively short time. However, there are a few incidents where breakdowns resulted in long unavailability but it was not possible to track the details due to poor documentation. Therefore, all maintenance data were captured from the maintenance team. Repair times follow the triangular distribution which uses three parameters: minimum, mode and maximum [6]. MTBF data follow the Uniform distribution which uses minimum and maximum parameters since it was not possible to establish the mode parameters. The most critical processes from maintenance point of view as well as their associated breakdown and repair data are shown in Table 4.

Table 4 Industrial case A breakdown and repair data

\begin{tabular}{|l|l|l|}
\hline Machine & MTBF (hours) & Repair time (hours) \\
\hline Buffing & Uniform $(160,192)$ & Triangle $(1,10,30)$ \\
\hline Cementing & Uniform $(160,192)$ & Triangle $(1,1.5,2)$ \\
\hline Building machine & Uniform $(320,384)$ & Triangle $(1,4,20)$ \\
\hline Enveloping & Uniform $(160,192)$ & Triangle $(1.5,2,2.5)$ \\
\hline Curing & Uniform $(1920,2304)$ & Triangle $(24,48,72)$ \\
\hline
\end{tabular}

Several assumptions were necessary to model PM. PM repair times are a third of CM repair times. In addition, PM is conducted internally and involves routine maintenance activities such as changing or topping oil, lubricating, cleaning, fixing electric wires ...etc. However, CM often involves spare parts and requires professionals from outside the factory. This will be reflected in higher maintenance costs for $\mathrm{CM}$ as can be expected. Table 5 presents both $\mathrm{CM}$ and PM costs in Local Currency Units (LCU). CM costs vary depending on the type of failure. For example, the buffing machine frequently breaks down as result of a broken gear which has to be fixed at an external workshop. The enveloping machine breakdown is due to a broken arm and can be fixed internally by replacing the part or using welding.

Table 5 CM and PM costs for case A

\begin{tabular}{|l|r|r|}
\hline Machine $\left(M \boldsymbol{c}_{\mathbf{i}}\right)$ & CM costs (LCU) & \multicolumn{1}{|c|}{ PM costs (LCU) } \\
\hline Buffing $\left(M c_{1}\right)$ & 3,200 & 300 \\
\hline Cementing $\left(M c_{2}\right)$ & 1,200 & 200 \\
\hline Building $\left(M c_{3}\right)$ & 450 & 150 \\
\hline Enveloping $\left(M c_{4}\right)$ & 200 & 50 \\
\hline Curing $\left(M c_{5}\right)$ & 3,500 & 400 \\
\hline
\end{tabular}

\subsection{Simulation Based Optimisation for Case A Maintenance System}

The framework described in Section 2.5 is followed step by step as follows: 
1. Define the scope of the optimisation: The assets in interest are already identified as shown in Table 4. Currently, the firm's management is interested in investigating maintenance strategies only. As the factory is located in an industrial area, spare parts are available locally from several suppliers. Investing in a warehouse for spare parts is not being considered. In addition, the management were not considering investing in creating more buffer spaces for Work In Progress.

2. Identify applicable maintenance strategies and policies: In addition to $\mathrm{CM}$, time-based PM is applicable for the critical machines. CBM will require investment and skilled labour which is not a possibility in the current situation.

3. Formulate the objective function: The two relevant objectives for this case are maximising the production throughput and minimising the maintenance cost. The maintenance cost function consists of $\mathrm{CM}$ and PM costs.

4. Define the decision variables: In addition to the maintenance strategy and the PM frequency for each machine, an additional decision variable from the maintenance resources group is considered. Up to two maintenance technicians costing each 24,000 LCU per year can be hired to assist with maintenance actions as opposed to the current situation where operators are conducting the maintenance tasks.

5. Define constraints: There is not sufficient knowledge to set the bounds for the PM frequency for each machine. Therefore, an estimate is made based on the minimum and maximum MTBF data. PM frequency bounds will be set to be between half the minimum MTBF and twice the maximum MTBF for each machine. Maintenance strategies $\left(M S_{i}\right)$ can be either 0 or 1 which represents $\mathrm{CM}$ and $\mathrm{PM}$ respectively. In addition, the variable $M S_{i}$ will be included in the bounds of PMfreqi to force it to equal to zero if the chosen maintenance strategy was $\mathrm{CM}$. Maintenance technicians can range between 0 and 2.

The optimisation problem can be formulated as follows:

Minimise: Maintenance Cost

Maximise: Production Throughput

Subject to:

$$
\begin{aligned}
& 80 *\left(M S_{1}\right)<P M \text { freq }_{1}<288 *\left(M S_{1}\right) \\
& 80 *\left(M S_{2}\right)<P M f r e q_{2}<288 *\left(M S_{2}\right) \\
& 160 *\left(M S_{3}\right)<P M \text { freq }_{3}<576 *\left(M S_{3}\right) \\
& 80 *\left(M_{4}\right)<P M \text { freq }_{4}<288 *\left(M S_{4}\right) \\
& 960 *\left(M_{5}\right)<P M \text { freq }_{5}<3456 *\left(M S_{5}\right) \\
& M S_{1}=0 \text { for } C M \text { or } 1 \text { for } P M \\
& M S_{2}=0 \text { for } C M \text { or } 1 \text { for } P M \\
& M S_{3}=0 \text { for } C M \text { or } 1 \text { for } P M \\
& M S_{4}=0 \text { for } C M \text { or } 1 \text { for } P M \\
& M S_{5}=0 \text { for } C M \text { or } 1 \text { for } P M
\end{aligned}
$$

$0<$ Maintenance technician $<2$ 
6. Select the optimisation algorithm: The framework suggests suitable optimisation algorithms based on a series of questions. The current optimisation problem is multi-objective. In addition, it requires global search. NSGA II is one of the options provided by the framework for similar problems. As Witness Optimizer does not include the required optimisation algorithm, GANetXL was connected to Witness as described in Section 2.

7. Set the simulation optimisation: The simulation run-length is set to two years. A variability analysis was conducted to establish the required number of replications. As can be seen from Figure 6, throughput begins to stabilise around the $8^{\text {th }}$ replication. However, when considering maintenance cost, the moving average starts to stabilise after the $13^{\text {th }}$ replication. Therefore, the number of replications will be set to 13 for this case.

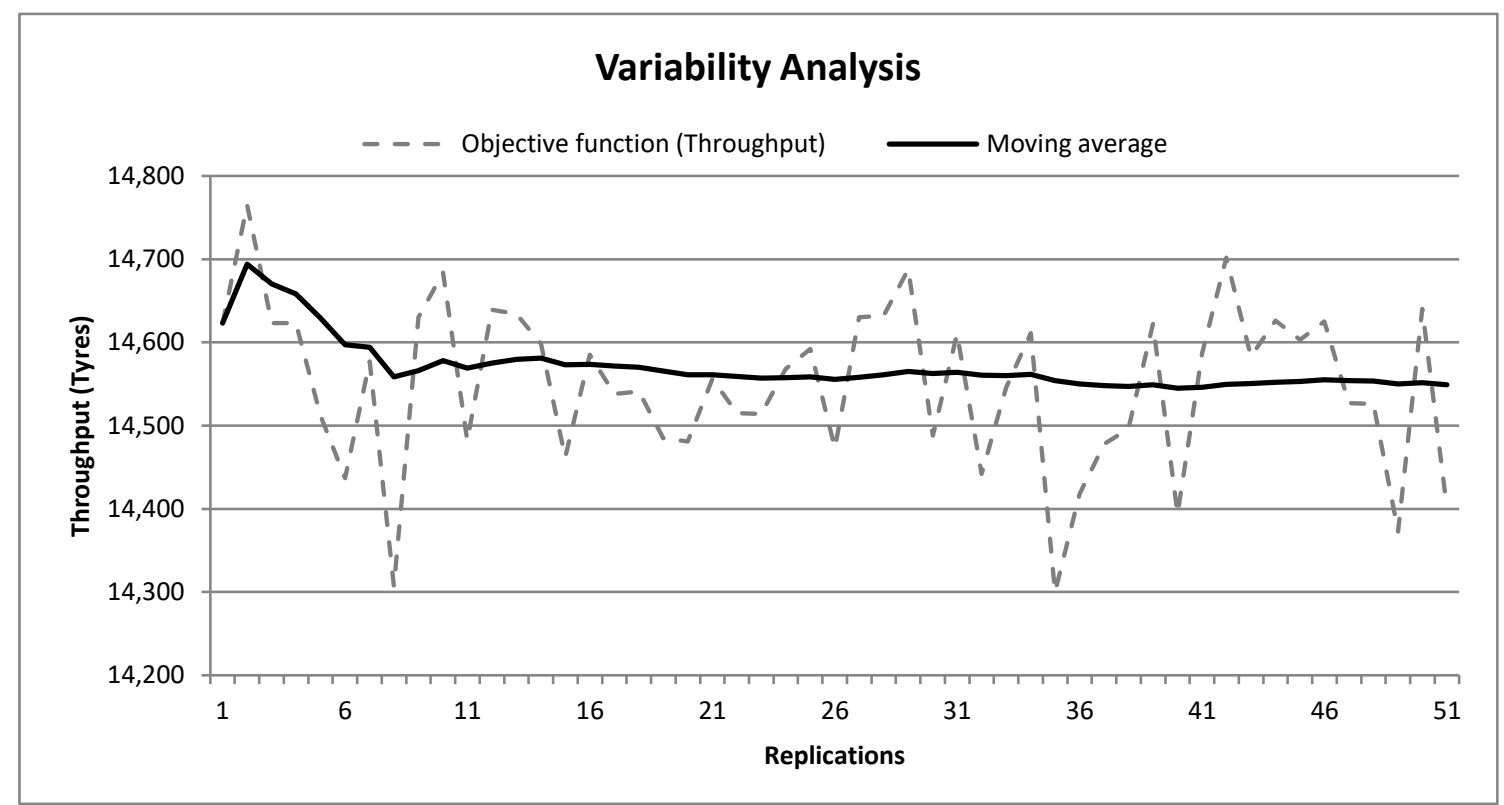

Figure 6 Variability analysis for case A simulation model considering throughput as an objective 


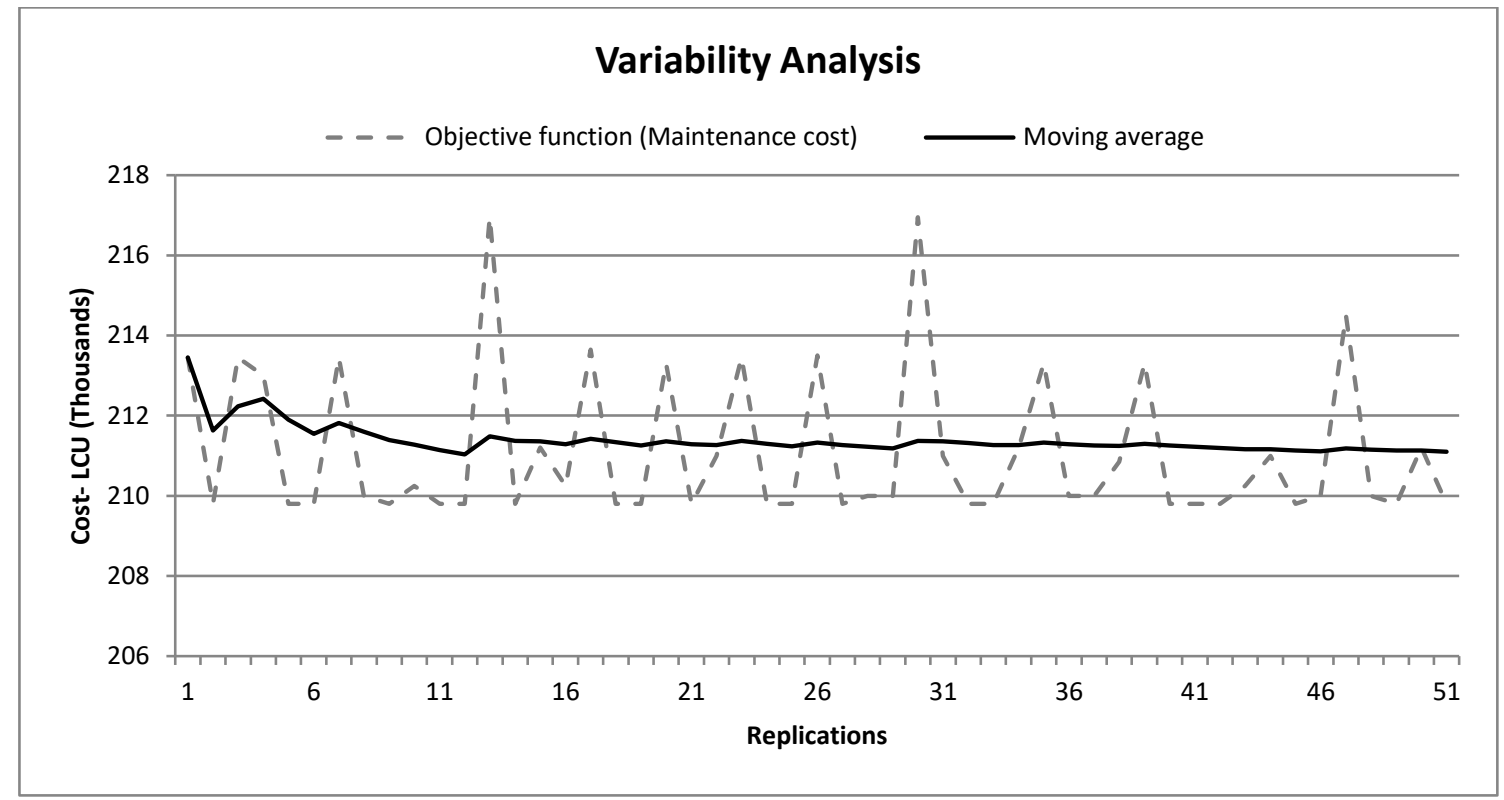

Figure 7 Variability analysis for case A simulation model considering maintenance cost as an objective

Similarly, to establish the required warm-up time, an analysis was conducted using Welch's Method. The moving average for production throughput and maintenance cost is plotted in Figure 8 and Figure 9 respectively. It can be concluded from both figures that 30 days is sufficient for the model to settle into steady state.

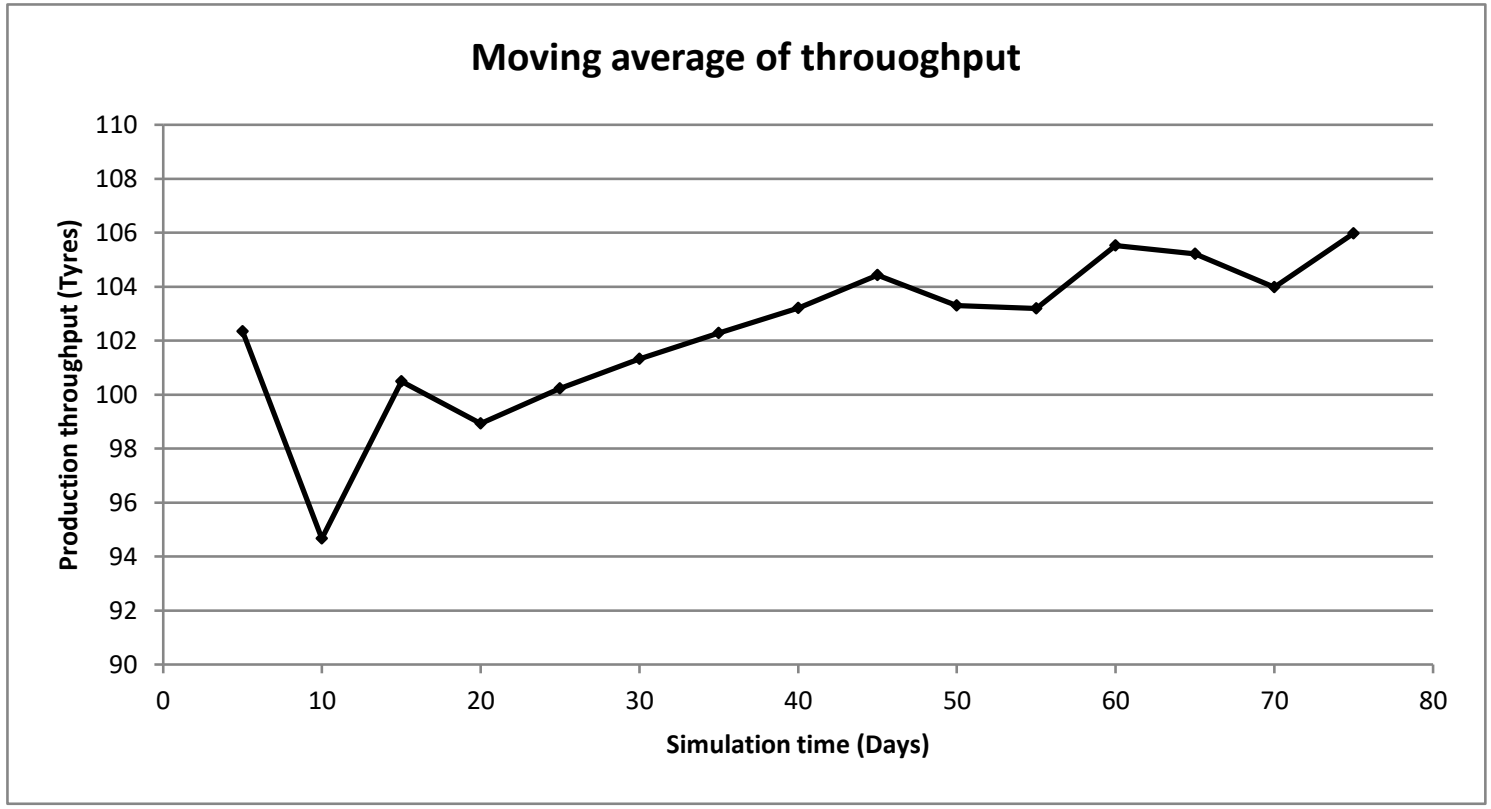

Figure 8 Warm-up analysis for case A simulation model considering throughput as an objective 


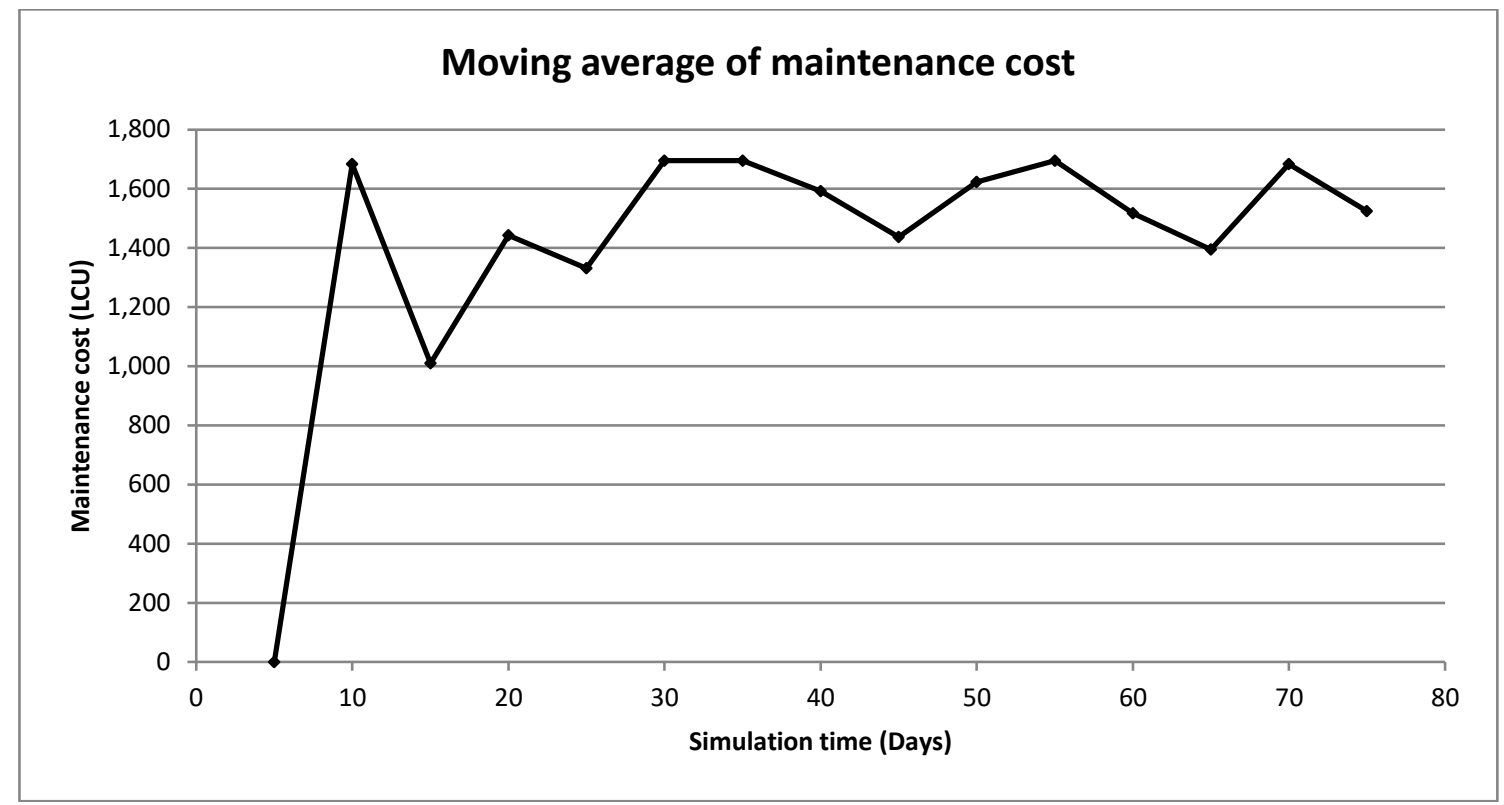

Figure 9 Warm-up analysis for case A simulation model considering maintenance cost as an objective

The simulation optimisation was run for a combination of population sizes and number of generations. Each combination was run using three different random seeds. Starting with a population size of 50 and 100 generations, the number of generations is increased gradually as long as GA is making progress. If no significant improvements in the results are apparent, the population size is set to 75 and then 100 and the process is updated. Only non-dominated solutions from the different random seeds were used to plot the data.

It is observed that none of the optimal solutions are close to the boundary set previously for decision variables. Therefore, there is no need to re-set the variables bounds and repeat the experiments.

8. Decision making: The current business environment is generally stable. MOO produces a number of non-dominated solutions. This provides flexibility to the decision maker since trade-off analysis can be made as the business environment changes.

Figure 10 presents the results for a population size of 50 . The results improved gradually while increasing the number of generations. However, the improvements in 400 generations were limited. It is interesting to observe that higher number of generations produce less spread and fewer solutions. It seems the Pareto front is quite narrow, so that as the algorithm converges we get fewer solutions. 


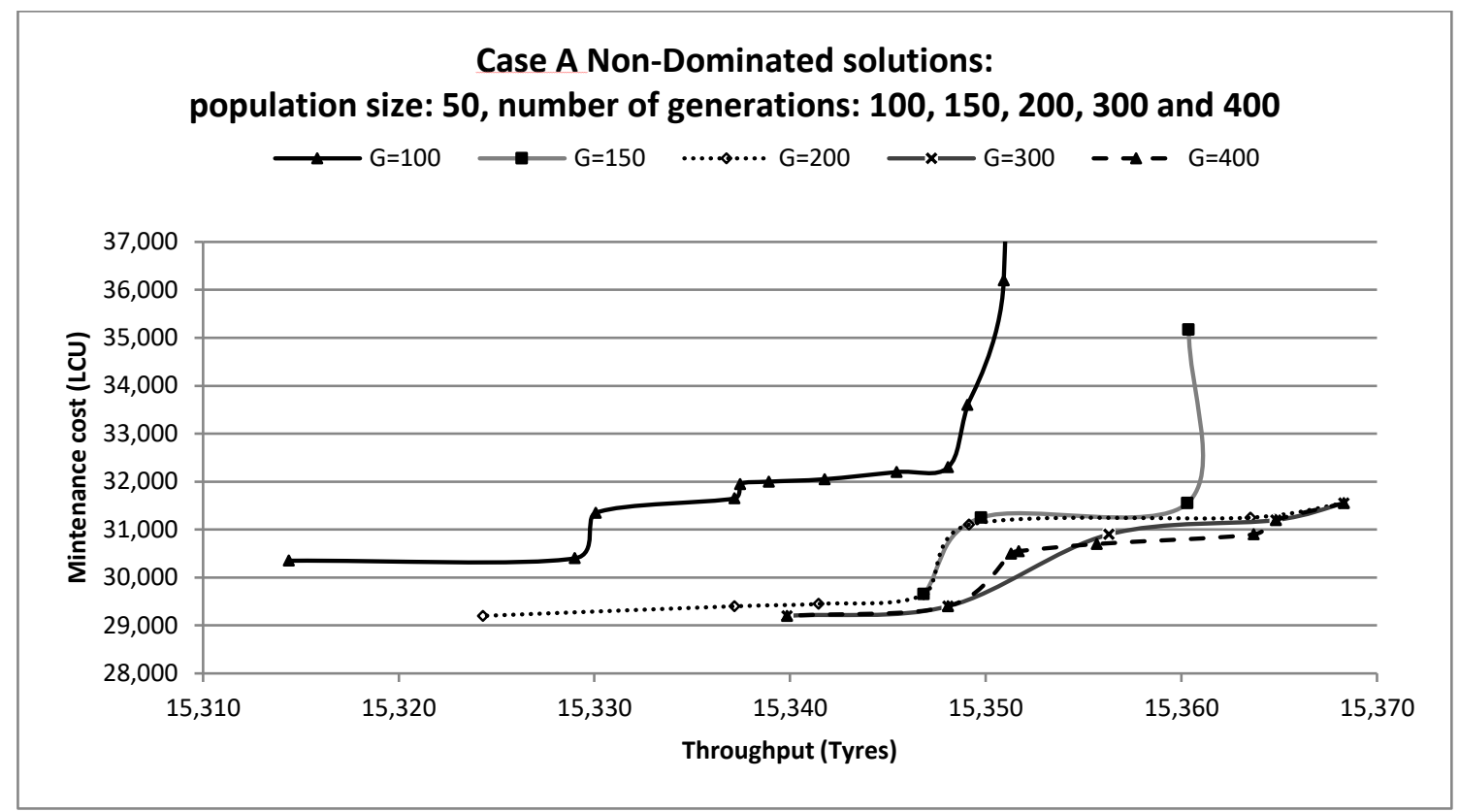

Figure 10 Case A Non-Dominated solutions: population size: 50, number of generations: 100, 150, 200, 300 and 400

The algorithm appeared to make less progress for different numbers of generations shown in Figure 11, because with this larger population the search had already achieved good results towards the final optimal set of solutions by only generation 100. Changes in the spread of solutions are also then small, confirming the existence of a narrow Pareto optimal set. Thus, in general population size 75 achieved noticeably better results than population size 50 .

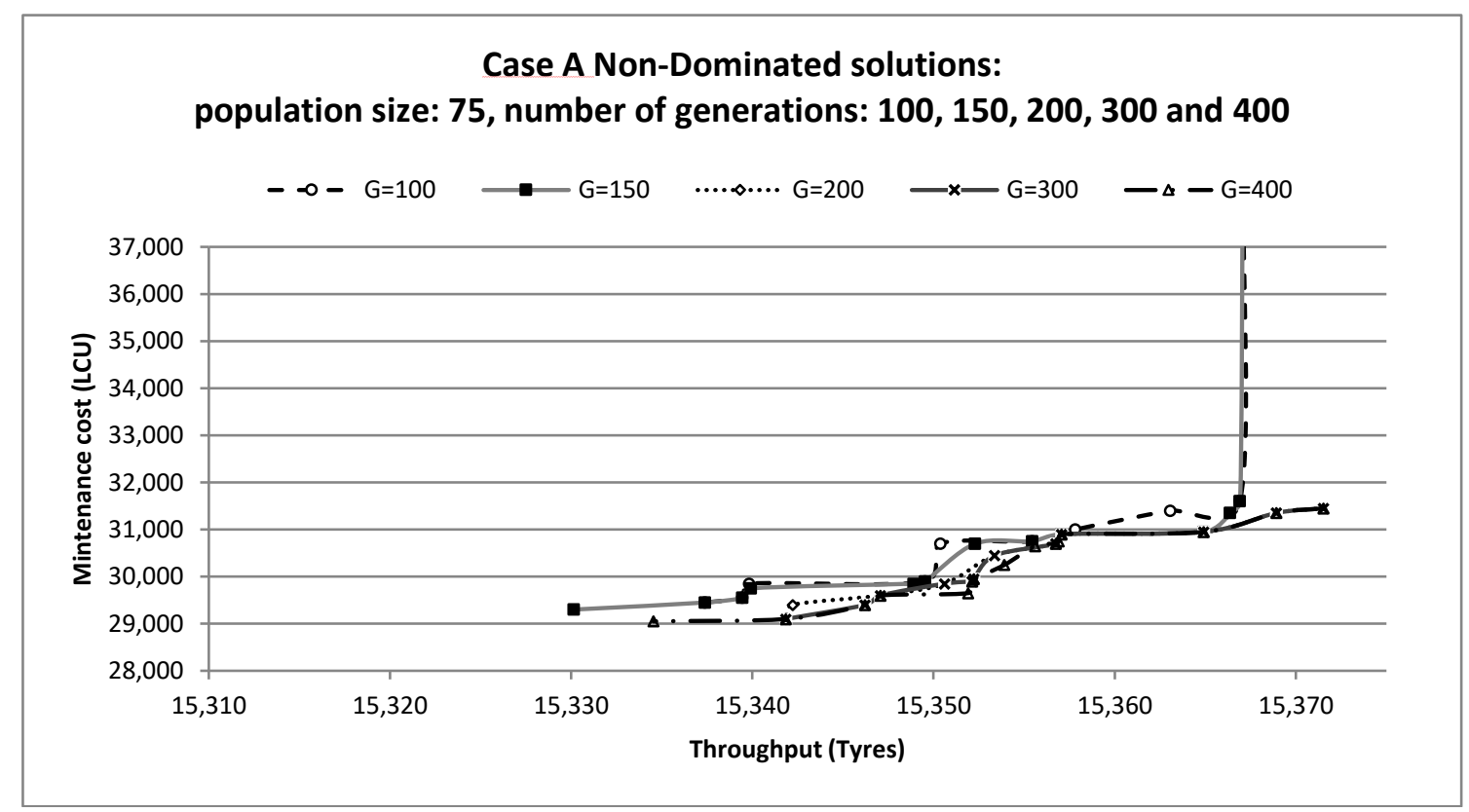

Figure 11 Case A Non-Dominated solutions: population size: 75, number of generations: 100, 150, 200, 300 and 400 
Population size 100 was run for 100,150 and 200 generations only due to the even smaller changes observed with increasing numbers of generations (see Figure 12). Interestingly the spread in solutions was now significantly less than that observed with population sizes of 50 and 75, probably reflecting the greater time now needed to develop solutions.

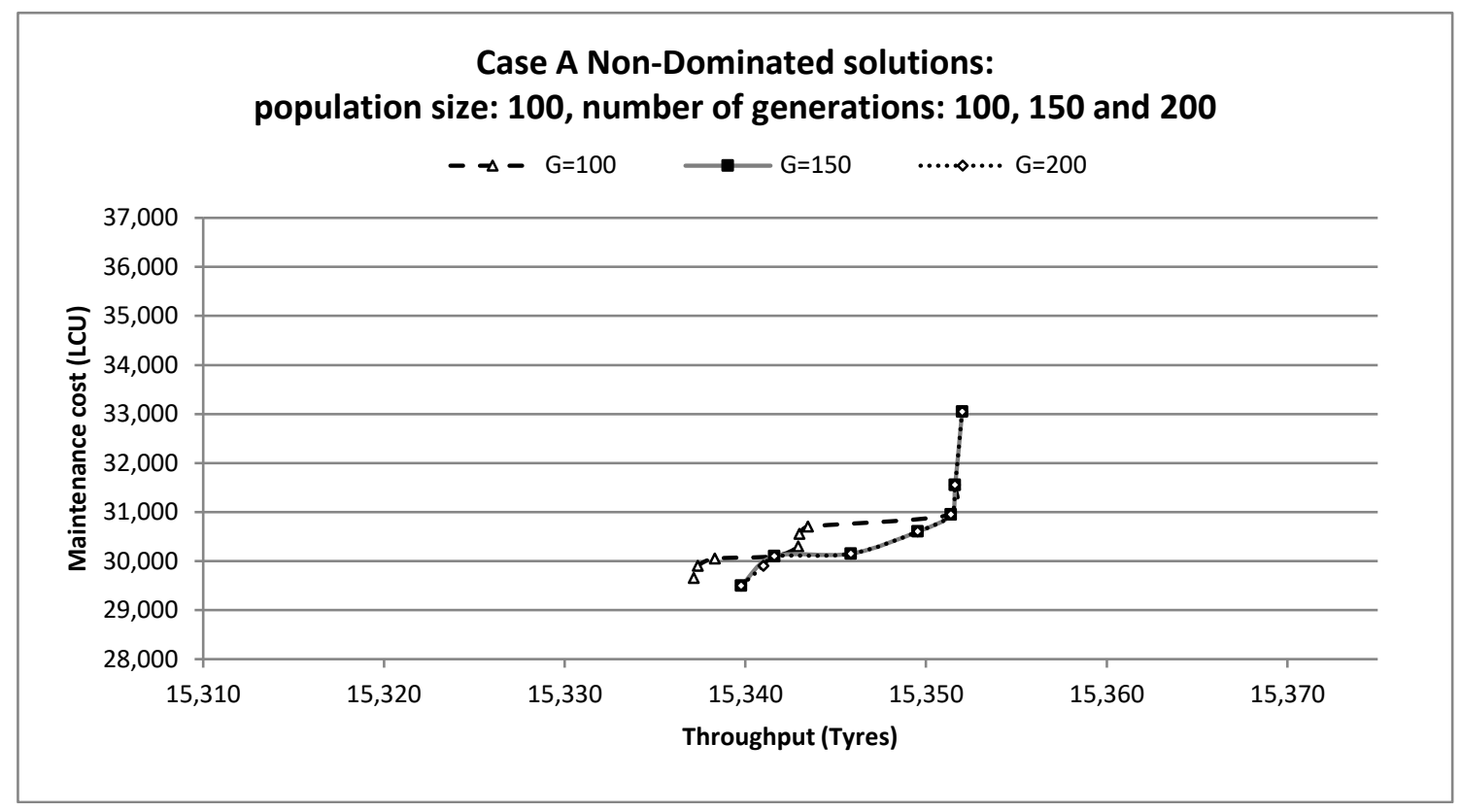

Figure 12 Case A Non-Dominated solutions: population size: 100, number of generations: 100, 150 and 200

Figure 13 shows the results of the three different population sizes for both 100 and 200 generations. It clearly illustrate the benefit of increasing population size from 50 to 75 , while it seems the largest population size of 100 needs more time/generations to develop results sufficiently 


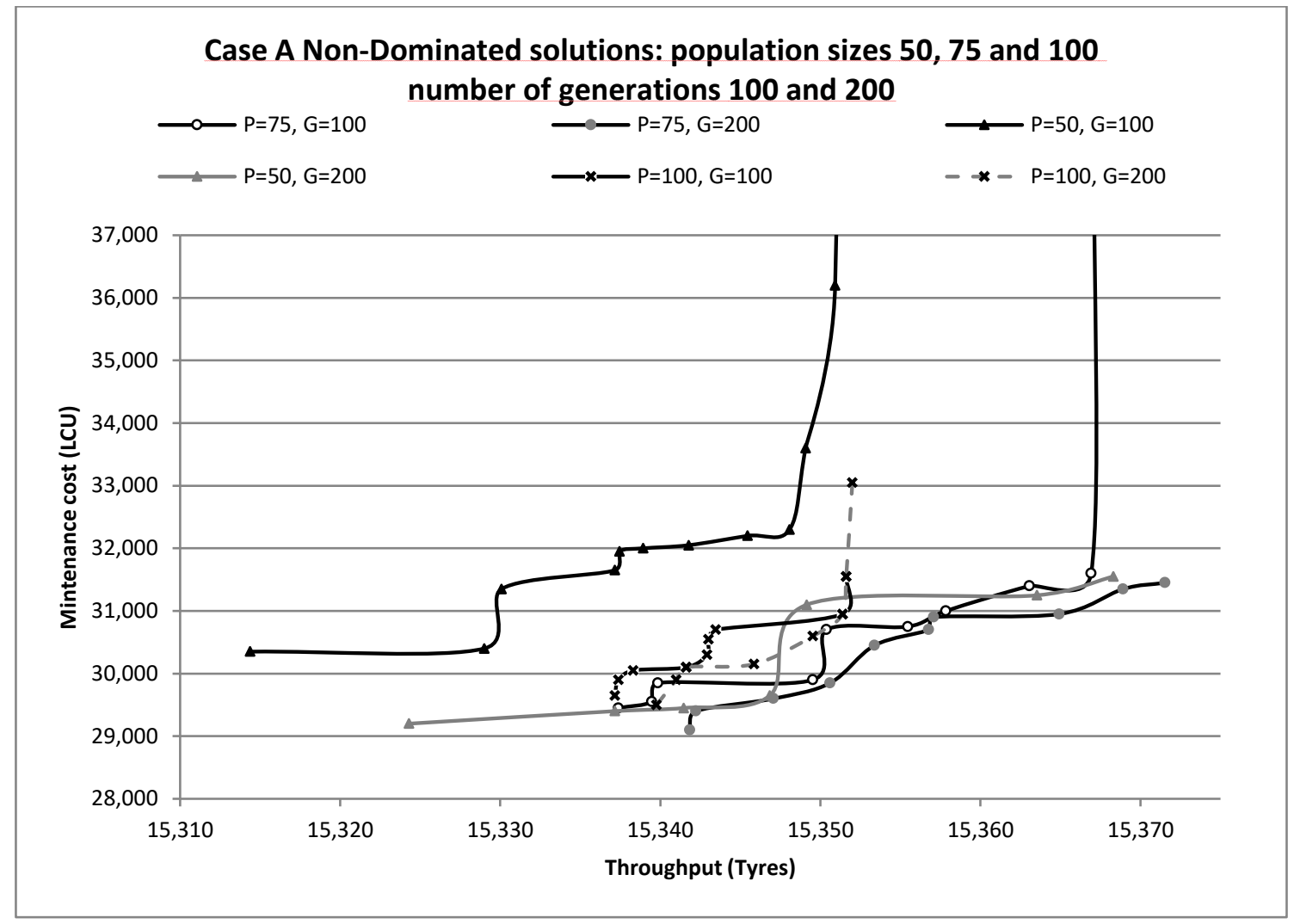

Figure 13 Case A Non-Dominated solutions: population sizes 50, 75 and 100 number of generations 100 and 200

All non-dominated solutions are plotted in Figure 14. The curve representing population size 75 and 400 generations appears to achieve the best solutions resulting in maximum production throughput and minimum maintenance cost. 


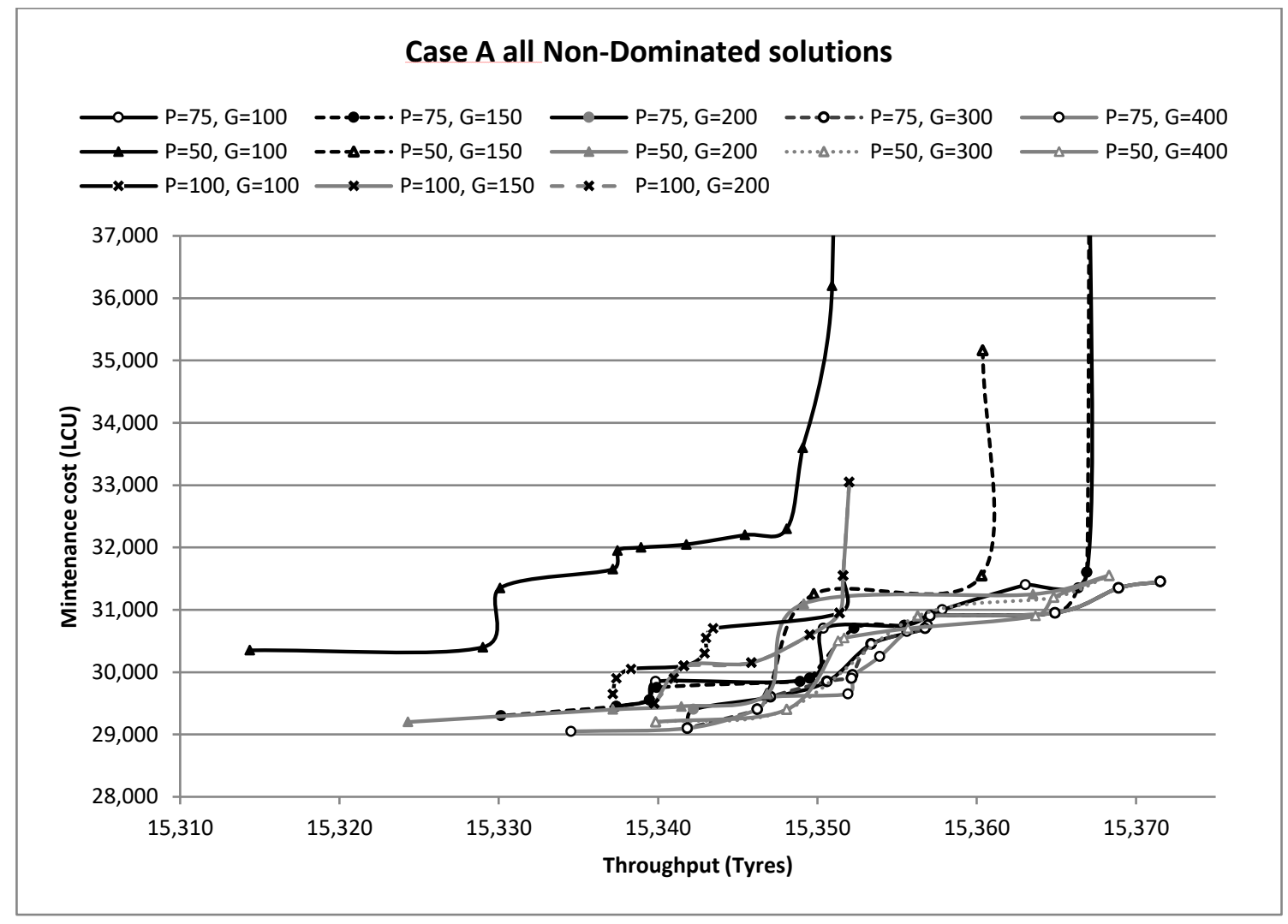

Figure 14 Case A all Non-Dominated solutions

The optimal solutions for the population size 75 and 400 generations are shown in Table 6. All the optimal solutions consider PM for all machines. In addition, no maintenance technicians are considered. Therefore, it can be concluded that PM is the optimum strategy for all machines and no additional specialised maintenance technicians are required at this stage. Selecting the optimum PMfreq from the set of non-dominated solutions depends on the business environment and whether investing more in maintenance can be justified by the increase in the production output. 
Table 6 Case A non-dominated solutions, population size: 75 , number of generations: 400

\begin{tabular}{|c|c|c|c|c|c|c|c|c|c|c|c|c|}
\hline \multicolumn{11}{|c|}{ Decision Variables } & \multicolumn{2}{|c|}{ Objectives } \\
\hline 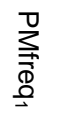 & $\begin{array}{l}\frac{0}{2} \\
\frac{1}{\mathbb{D}} \\
\frac{D}{N}\end{array}$ & $\begin{array}{l}\frac{0}{2} \\
\stackrel{\vec{\Phi}}{\frac{D}{\omega}}\end{array}$ & 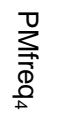 & $\begin{array}{l}\text { Da } \\
\text { 竞 } \\
\text { o }\end{array}$ & ळ) & $\underset{N}{3}$ & $\underset{\omega}{\vec{\omega}}$ & $\frac{3}{\infty}$ & 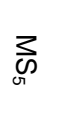 & 3 & $\begin{array}{c}\text { Throughput } \\
\text { (Tyres) }\end{array}$ & Cost (LCU) \\
\hline 158 & 159 & 313 & 158 & 1,884 & 1 & 1 & 1 & 1 & 1 & 0 & $15,334.54$ & $29,050.00$ \\
\hline 158 & 159 & 313 & 156 & 1,884 & 1 & 1 & 1 & 1 & 1 & 0 & $15,341.85$ & $29,100.00$ \\
\hline 160 & 159 & 314 & 135 & 1,895 & 1 & 1 & 1 & 1 & 1 & 0 & $15,345.69$ & $29,400.00$ \\
\hline 160 & 154 & 306 & 145 & 1,911 & 1 & 1 & 1 & 1 & 1 & 0 & $15,347.08$ & $29,600.00$ \\
\hline 160 & 158 & 282 & 147 & 1,903 & 1 & 1 & 1 & 1 & 1 & 0 & $15,351.92$ & $29,650.00$ \\
\hline 160 & 154 & 274 & 145 & 1,903 & 1 & 1 & 1 & 1 & 1 & 0 & $15,352.15$ & $29,900.00$ \\
\hline 160 & 147 & 306 & 147 & 1,903 & 1 & 1 & 1 & 1 & 1 & 0 & $15,352.23$ & $29,950.00$ \\
\hline 160 & 158 & 314 & 159 & 1,405 & 1 & 1 & 1 & 1 & 1 & 0 & $15,353.92$ & $30,250.00$ \\
\hline 160 & 158 & 314 & 159 & 1,389 & 1 & 1 & 1 & 1 & 1 & 0 & $15,355.62$ & $30,650.00$ \\
\hline 159 & 159 & 318 & 154 & 1,389 & 1 & 1 & 1 & 1 & 1 & 0 & $15,356.77$ & $30,700.00$ \\
\hline 160 & 159 & 274 & 157 & 1,407 & 1 & 1 & 1 & 1 & 1 & 0 & $15,356.92$ & $30,750.00$ \\
\hline 160 & 154 & 274 & 159 & 1,407 & 1 & 1 & 1 & 1 & 1 & 0 & $15,357.08$ & $30,900.00$ \\
\hline 159 & 159 & 270 & 152 & 1,405 & 1 & 1 & 1 & 1 & 1 & 0 & $15,364.92$ & $30,950.00$ \\
\hline 160 & 159 & 274 & 143 & 1,391 & 1 & 1 & 1 & 1 & 1 & 0 & $15,368.92$ & $31,350.00$ \\
\hline 160 & 154 & 274 & 147 & 1,389 & 1 & 1 & 1 & 1 & 1 & 0 & $15,371.54$ & $31,450.00$ \\
\hline
\end{tabular}

\section{Industrial Case B}

\subsection{Description of Factory B}

Industrial case B is held in a large petrochemical company. Its products include: Aromatics, Acetic Acid, Purified Terephthalic Acid (PTA) and Bottle Grade Chips (PET) as illustrated in Figure 15.

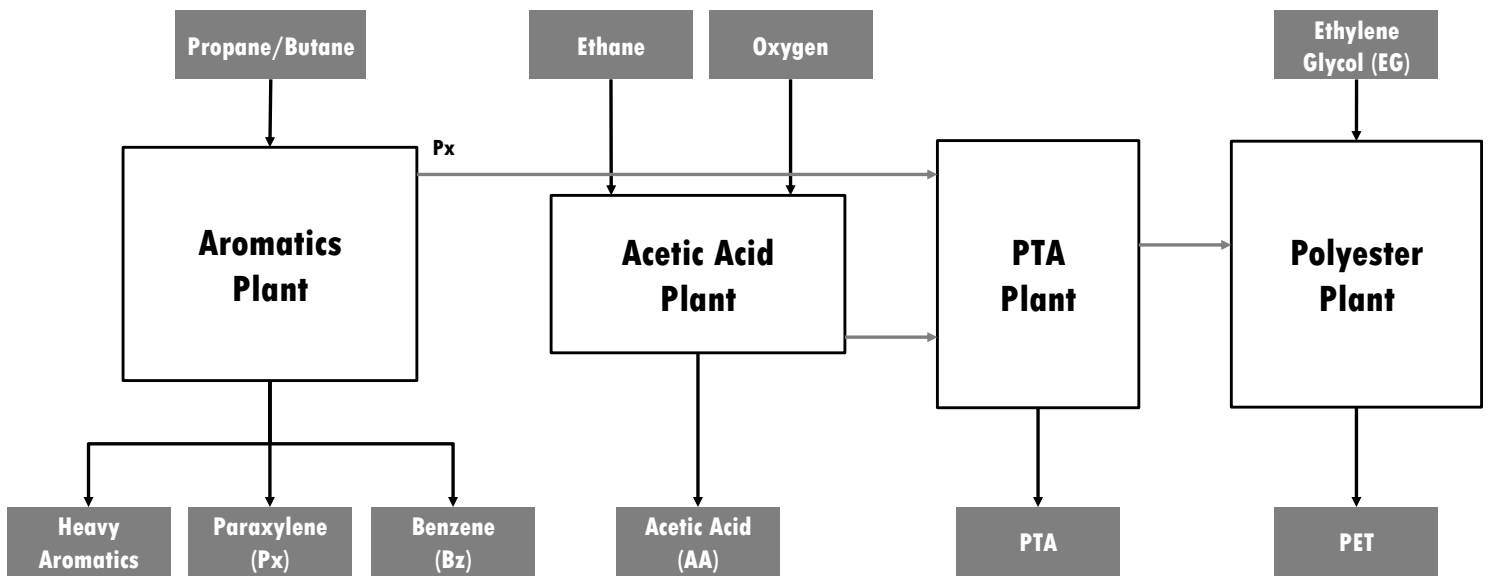

Figure 15 Plants in industrial case $B$

Detailed manufacturing data and accurate maintenance records are continuously updated in the SAP system. However, condition monitoring data are held separately in an asset management software. The focus of the current study is on one production line in the Polyester Plant, namely, Solid State Polycondensation (SSP) line. Polyester is formed by the polycondensation of 
PTA and Ethylene Glycol (EG) in a continuous manner using specialised catalyst in a series of reactors. The manufacturing process in SSP is illustrated in Figure 16.

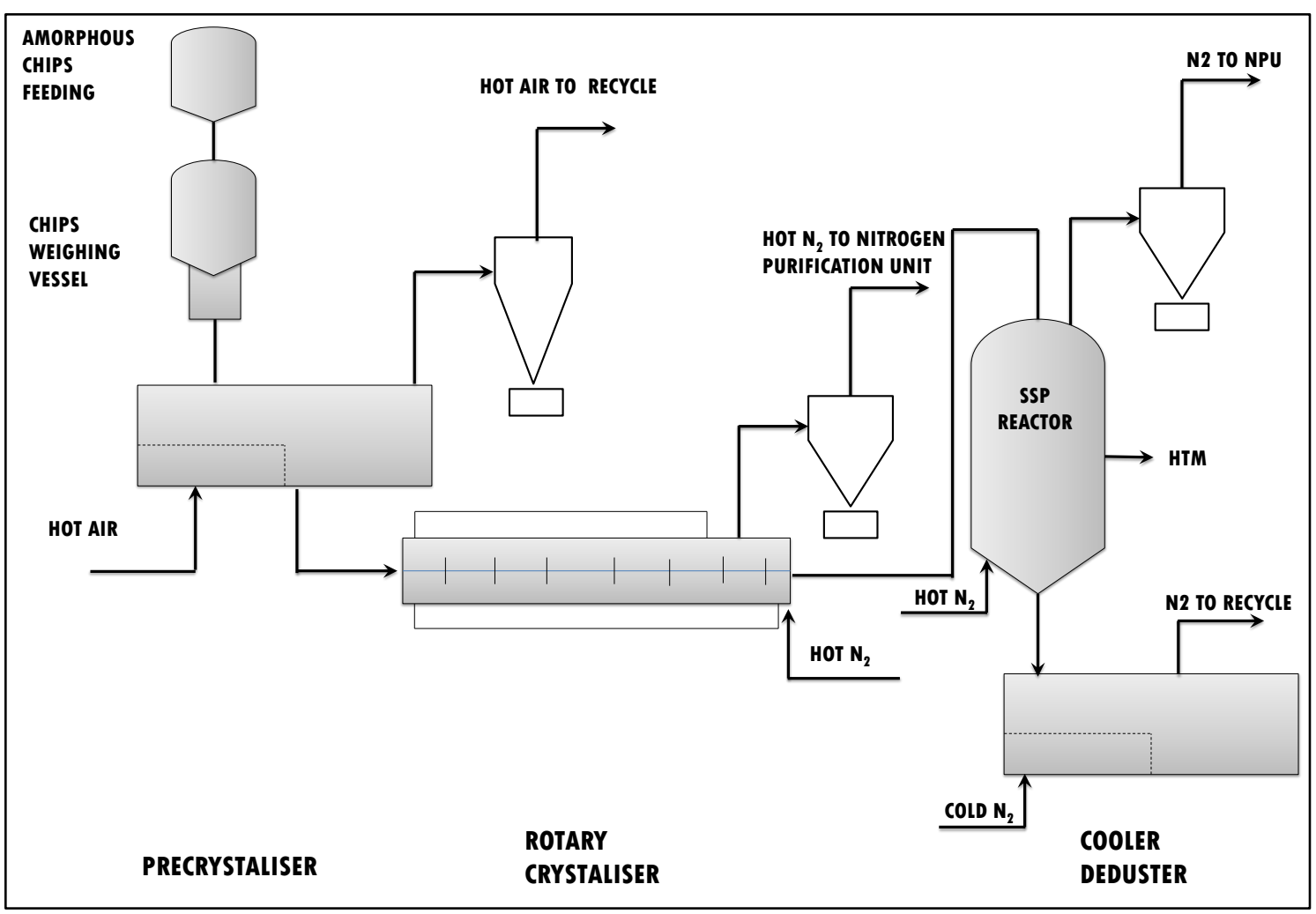

Figure 16 SSP flow diagram

Four main processes are involved as follows:

1. Pre Crystallisation:

- Amorphous chips from the silo are conveyed via pneumatic conveying \& rotary feeder into buffer vessels in which throughput of SSP is controlled by loss in weight system

- Chips are partially crystallised in the fluidised bed with a closed loop hot air system

- Pre Crystallised chips are fed into purge vessel where air is purged off with hot nitrogen

2. Crystallisation

- Chips are heated \& further crystallised in the Rotary Crystalliser

- Hot pure Nitrogen from Nitrogen Purification Unit (NPU) is passed through the crystalliser to separate Oligomer, Acetaldehydes \& moisture etc.

3. Solid State Polymerisation Reactor

- Chips from the Crystalliser are fed to SSP reactor via a vertical tube

- The partially crystallised chips are subjected to high temperature treatment in $\mathrm{O}_{2}$ and $\mathrm{H}_{2} \mathrm{O}$ free environment

- Removal of volatile impurities $\left(\mathrm{H}_{2} \mathrm{O}, \mathrm{EG}\right.$ etc.) is accomplished by diffusion to chips surface and carried out by hot pure nitrogen stream 
4. Cooling and de-dusting: The hot chips from reactor are cooled and de-dust for bagging

The residence time for fluids in each stage is shown in Table 7.

Table 7 Residence time for fluids in each stage

\begin{tabular}{|l|l|l|}
\hline & Stage & Residence time (hours) \\
\hline 1 & Pre Crystallisation & Uniform $(0.33,0.5)$ \\
\hline 2 & Crystallisation & Uniform $(0.5,1.0)$ \\
\hline 3 & Solid State Polymerisation Reactor & Uniform $(10,20)$ \\
\hline 4 & Cooling and de-dusting & Uniform $(0.67,0.83)$ \\
\hline
\end{tabular}

As fluid is continuously moving in the production line, if one machines breaks down, the whole line will be stopped. In addition, if the production line is stopped continuously for two hours or more, it has to be drained. Therefore, all machines will scrap the fluids. The simulation layout is shown in Figure 17.

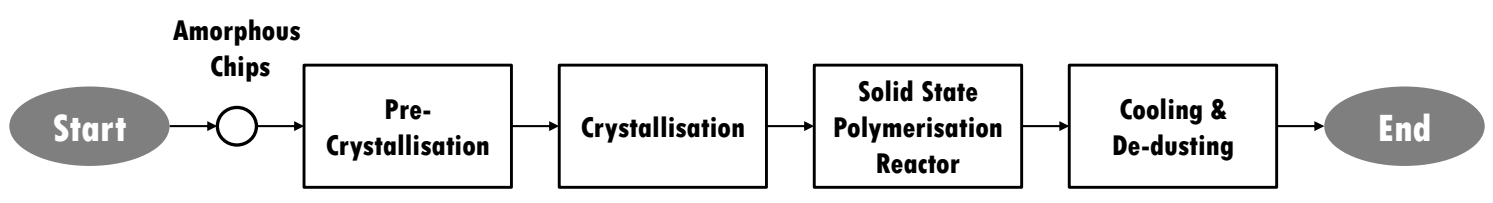

Figure 17 Case B simulation layout

\subsection{Maintenance Operations}

A range of maintenance strategies are applied including CM, OM and CBM. Table 8 shows CM and OM data. The standard rate for a labour hour is 144 Local Currency Units (LCU). However, OM costs 66\% less since it can occur when the asset has just been maintained. An additional cost of 5,000 LCU is incurred whenever $\mathrm{CM}$ occurs to reflect the fact that unscheduled breakdowns can result in relocation of maintenance and operation resources. CBM requires an investment of 50,000 per machine to cover the costs of required equipment, software, support and training. Each scheduled inspection costs $432 \mathrm{LCU}$ which includes taking the measurement and conducting the required analysis.

Table $8 \mathrm{CM}$ and OM data for case B

\begin{tabular}{|c|c|c|c|}
\hline Asset & MTBF & CM Repair time & OM repair time \\
\hline Pre-Crystalliser & Weibull $(0.586,598)$ & $\begin{array}{l}\text { 1/ Gamma (0.564, } \\
0.391)\end{array}$ & $\begin{array}{l}\text { 1/ Beta (0.744, } \\
14.6)\end{array}$ \\
\hline Crystalliser & Gamma $(0.61,3830)$ & $\begin{array}{l}\text { 1/ Gamma (0.92, } \\
0.309)\end{array}$ & $\begin{array}{l}\text { Triangular }(1,12, \\
180)\end{array}$ \\
\hline Reactor & Weibull $(0.676,969)$ & $\begin{array}{ll}1 / & \text { Beta } \\
1.22)\end{array}$ & $\begin{array}{l}\text { Triangular }(1,8, \\
1080)\end{array}$ \\
\hline Cooling & Gamma $(0.563,3350)$ & $\begin{array}{l}1 / \text { Beta } \quad \text { (0.529, } \\
1.99)\end{array}$ & $\begin{array}{l}\text { Triangular }(1,28 \text {, } \\
240)\end{array}$ \\
\hline
\end{tabular}

The condition of each machine is modelled according to the data presented in Table 9. Inspections are conducted while the production line is operated. 
Whenever a maintenance action occurs on a machine, the condition is reset to the normal operation level.

Table 9 Condition monitoring data for case B

\begin{tabular}{|l|l|l|l|}
\hline Asset & $\begin{array}{l}\text { Probability of no } \\
\text { change in the } \\
\text { condition indicator }\end{array}$ & $\begin{array}{l}\text { Asset degradation (PK } \\
\mathbf{m m} / \mathbf{S e c}) / 5 \text { days }\end{array}$ & $\begin{array}{l}\text { Normal operation } \\
\text { level (PK mm/Sec) }\end{array}$ \\
\hline Pre-Crystalliser & $63 \%$ & $\begin{array}{l}\text { Triangular }(0.103,0.207, \\
0.413)\end{array}$ & 0.43 \\
\hline Crystalliser & $84 \%$ & 0.1 & 2.65 \\
\hline Reactor & $53 \%$ & $\begin{array}{l}\text { Triangular }(0.105,0.209, \\
5.018)\end{array}$ & 1.85 \\
\hline Cooling & $15 \%$ & $\begin{array}{l}\text { Triangular }(0.102,0.1021, \\
0.562)\end{array}$ & 1.85 \\
\hline
\end{tabular}

\subsection{Simulation Based Optimisation for Case B Maintenance System}

The framework described in Section 2.5 is followed step by step as follows:

1. Define the scope of the optimisation: Discussions with both production and maintenance teams resulted in the identification of the critical assets as shown in Table 7. Spare parts policies are decided centrally for the whole corporation. Therefore, it is not possible to alter spare parts parameters. In addition, it is not possible to invest in extra buffer systems. As a result, the optimisation scope will be limited to the maintenance system only.

2. Identify applicable maintenance strategies and policies: In addition to considering $\mathrm{CM}$ as a maintenance strategy, $\mathrm{OM}$ is considered since the production line is continuous and the opportunity of a breakdown can be seized to conduct maintenance actions. CBM with periodic inspections is applicable and is considered as a possible maintenance strategy. It appears that CBM with periodic inspections is more efficient than time-based PM. This is because in the latter, maintenance is preformed regularly forcing a shutdown without considering the condition of assets. Inspections in CBM are conducted without affecting the operational status of the production line. The production line will be stopped to execute CBM only when it is necessary. Therefore, time-based PM is not considered in this case.

3. Formulate the objective function: Maximising production throughput is the main concern for the company. However, this objective has to be achieved at the minimum possible cost. Maintenance costs include the costs of conducting $\mathrm{CM}, \mathrm{OM}$ and $\mathrm{CBM}$.

4. Define the decision variables: The decision variables suggested by the framework are: the maintenance strategy for each machine, the inspection frequency for each machine and the CBM threshold for each machine. No other decision variables are required for this problem.

5. Define constraints: $O M_{i}, C M_{i}$ and $C B M_{i}$ are defined as decision variables that represent the selected maintenance strategy for each machine. The value 1 means the maintenance strategy is selected whereas the value 0 means the maintenance strategy is not selected. Since only one 
maintenance strategy can be selected for each machine at any time, the following constraint needs to be added: $O M_{i}+C M_{i}+C B M_{i}=1$

Inspections bounds can be set to take place between 15 and 60 days. CBM threshold values range between the normal operation level and the maximum vibration level. The optimisation problem can be defined as follows:

Minimise: Maintenance Cost

Maximise: Production Throughput

Subject to:

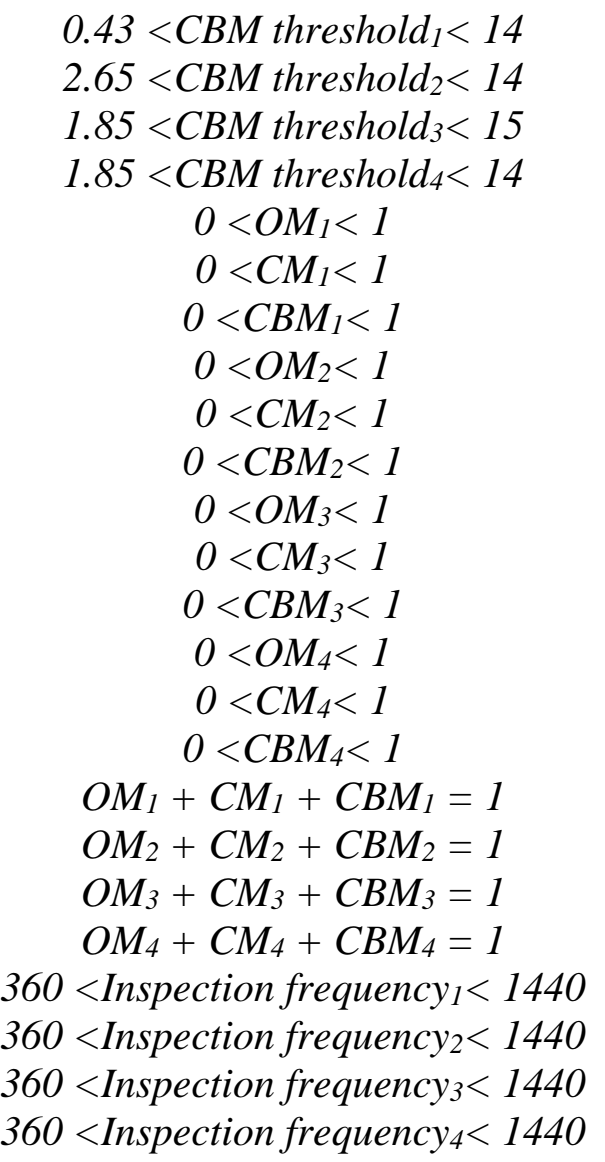

$O M_{i}, C M_{i}, C B M_{i}$ and Inspection frequency $y_{i}$ are integers

6. Select the optimisation algorithm: Following the framework flowchart results in selecting multi-objective optimisation as well as a problem that requires global search. As a result, NSGA II is one of the alternative optimisation algorithms that are suitable for this type of problem. It selected to solve the optimisation problem in hand.

7. Set the simulation optimisation: The simulation run-length is 3 years. Variability analysis similar to the one described earlier in Case A was conducted considering throughput and maintenance cost as objectives. It revealed that the objective function stabilise after 11 replications. Therefore, it will be selected as the number of replications for this case. Likewise, warm-up analysis on both throughput and maintenance cost revealed that 25 
days are sufficient for the simulation model to reach a steady state since changes in the moving average are minimal after this period. NSGA II was run for a combination of population sizes and generations.

8. Decision making: It is interesting to observe that NSGA II produced a limited number of non-dominated solutions as shown in Figure 18. In fact, instead of the expected non-dominated front, the optimisation resulted in a single optimal solution (population size 100 and 100 generations). This could be an indication that maintenance cost and throughput are not conflicting objectives in this case. It is also interesting to observe that increasing the number of generations improved the results slightly for population size 50 while it did not improve the results at all for population sizes 75 and 100 . In other words, increasing the number of generations for population size 50 from 150 to 200 did not yield better solutions. Similarly, increasing the number of generations from 100 to 150 and then subsequently to 200 for population sizes 75 and 100 did not result in improved solutions.

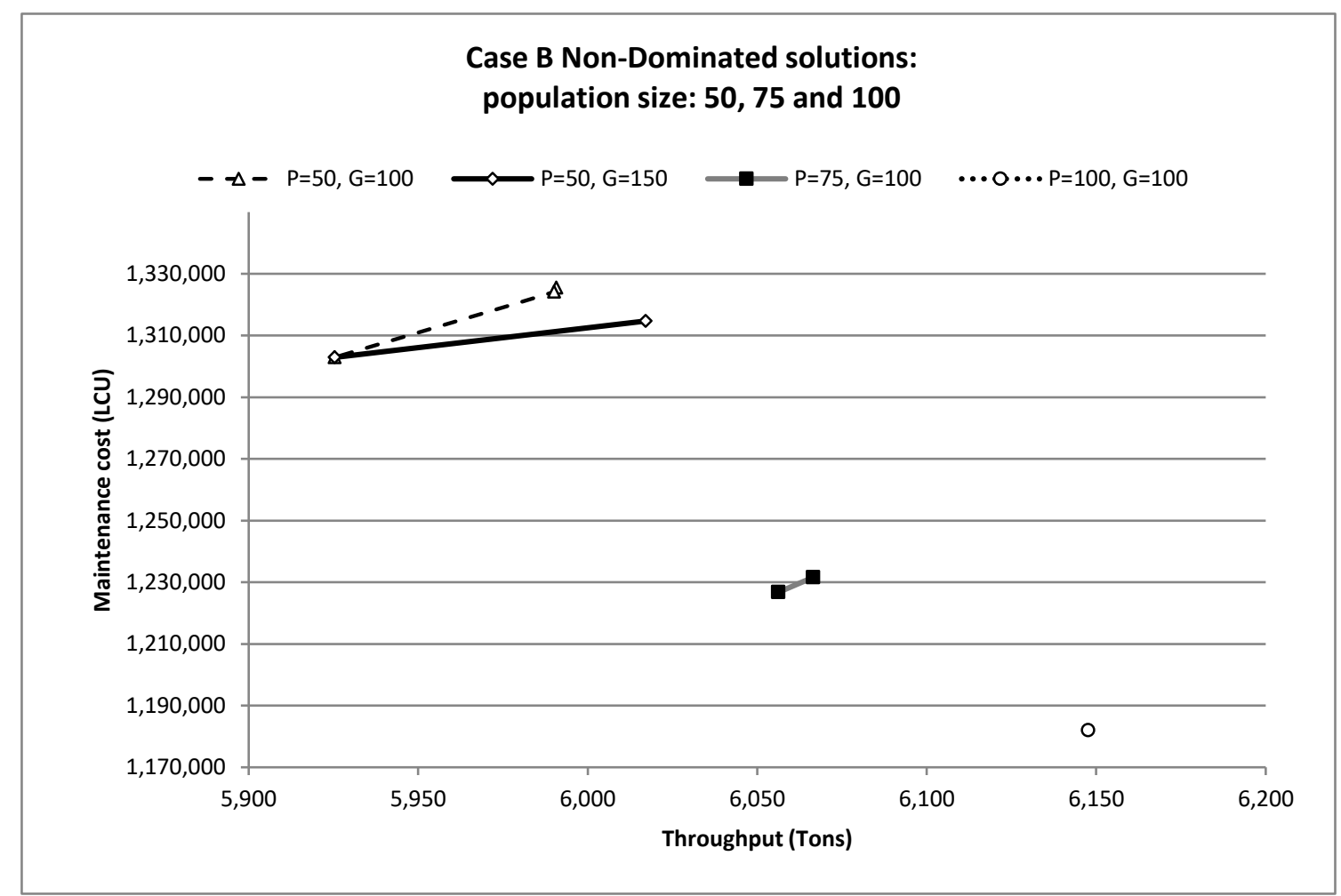

Figure 18 Case B all Non-Dominated solutions

The optimal solution (population size 100 and 100 generations) is presented in Table 10. From this data, we can see that the optimum maintenance strategy is different for each machine. The optimum maintenance strategy for the pre-crystallisation process is CBM. An associated periodic inspection is suggested to be scheduled every 783 hours and CBM to be conducted if the vibration level exceeds $2.41 \mathrm{PK} \mathrm{mm} / \mathrm{Sec}$. OM is the optimum maintenance strategy for the crystallisation process. $\mathrm{CM}$ is the optimum strategy for both the reactor and the cooling processes. 
Table 10 Optimal solution for case B

\begin{tabular}{|c|c|c|}
\hline \multirow{20}{*}{ Decision variables } & CBM threshold ${ }_{1}$ & 2.41 \\
\hline & CBM threshold 2 & 6.11 \\
\hline & CBM threshold 3 & 13.39 \\
\hline & CBM threshold $_{4}$ & 6.24 \\
\hline & OM1 & 0 \\
\hline & CM1 & 0 \\
\hline & CBM1 & 1 \\
\hline & OM2 & 1 \\
\hline & $\mathrm{CM} 2$ & 0 \\
\hline & CBM2 & 0 \\
\hline & OM3 & 0 \\
\hline & CM3 & 1 \\
\hline & CBM3 & 0 \\
\hline & OM4 & 0 \\
\hline & CM4 & 1 \\
\hline & CBM4 & 0 \\
\hline & Inspection frequency ${ }_{1}$ & 783 \\
\hline & Inspection frequency 2 & 1,434 \\
\hline & Inspection frequency ${ }_{3}$ & 709 \\
\hline & Inspection frequency 4 & 1,037 \\
\hline \multirow{2}{*}{ Objectives } & Cost (LCU) & $1,181,926.31$ \\
\hline & Throughput (Tons) & $6,147.61$ \\
\hline
\end{tabular}

It is surprising to see that $\mathrm{CM}$ is the optimum maintenance strategy for two processes while more advanced maintenance strategies are available. This could be attributed to the high expenses associated with the installation of CBM which significantly affects the cost function. As this production line is continuous, OM can result in unnecessary delays for shutdowns. However, it appears that aged-based or time-based PM - implying periodic shutdowns could prove beneficial for this type of production line.

Although the decision variables CBM threshold and inspection frequency are only significant if the selected maintenance strategy is CBM, it is still considered in the solution string by the optimisation algorithm even if the selected maintenance strategy is $\mathrm{CM}$ or $\mathrm{OM}$. The current optimisation engine requires all decision variables to be defined at the same level. It is not possible to include a given decision variable only if another decision variable has certain values. As a result, in some runs, the optimisation algorithm would change the parameters of a maintenance strategy that is not selected resulting in wasting time by conducting meaningless simulation optimisation cycles.

\section{Discussion}

The objective of this paper is to investigate the optimisation of two empirical maintenance systems. The maintenance systems varied in terms of sector, size, number of manufacturing processes and level of maintenance documentation.

Very little was found in prior studies on discussing the scope of optimisation, investigating applicable maintenance strategies or formulating the optimal 
problem [5]. However, in the current research, the simulation-based optimisation framework [13] guided the process of connecting the simulation model to the optimisation engine. Application of the framework resulted in proper discussion of optimisation scope, applicable maintenance strategies and optimal problem formulation for each case. The methodology has been shown to work for two very different real industrial maintenance processes and that separate lessons have been learnt from each, which can inform subsequent applications to others, while revealing different restrictions associated with interdependencies of key objectives.

While the majority of prior studies focused on optimising the parameters of a given maintenance strategy $[5 ; 14]$, the results of industrial case $B$ is one of the first to show the possibility of optimising maintenance strategies resulting in a different maintenance strategy for each asset. Since a change in one element of the simulation model such as buffer capacity or PMfreq for any asset in the system might affect the maintenance performance, it is difficult to assume an optimum maintenance strategy for any given asset. Therefore, it is suggested to evaluate all applicable maintenance strategies for critical assets in the system. The modelling of industrial systems involving various maintenance strategies was made possible using the modelling approach suggested by the authors [7].

Production throughput and maintenance cost were found to be conflicting objectives in case A. Conducting PM in shorter frequencies seems to increase the maintenance cost and increase the throughput at the same time. Similar trade-off solutions between cost and availability [11; 12], cost and reliability [15] and cost and profit [16] can be found in literature. One unanticipated finding from the results of case $B$ was that maintenance cost and production throughput might be non-conflicting in some cases. The two objectives appear to be initially conflicting, but as the solutions converge, only one non-dominated solution emerged indicating that objectives might be in fact non-conflicting [17].

In NSGA II, one may expect better solutions as the population size is increased. However, the results of this study shows that increasing the population size may lead to worse solutions. Population sizes 50 and 75 achieved better solutions in case A compared to population size 100. It is difficult to explain this result, but it might be related to the fact that each optimisation problem requires a certain population size and number of generations to achieve the best balance between diversity and conversion while considering present limitations such as time and computation expenses. In general, it is understood that increasing the population size leads to better diversification in solutions whereas running the algorithm for more generations leads to better conversion to the optimal front [17]. In this case, it is possible that larger population sizes negatively affected the progression of the algorithm towards the Pareto front by attempting to achieve better diversity.

While investigating multiple maintenance strategies, the optimisation algorithm might search in a useless space because some variables depend on the choice of maintenance strategy. For example, in case B inspection frequency and CBM threshold are relevant only if the selected maintenance strategy is CBM. However, NSGA II would search for inspection frequency and CBM threshold 
for an asset even if the selected maintenance strategy was $\mathrm{CM}$ or OM. This may reduce the algorithm's efficiency and result in running unnecessary simulations.

One of the issues that emerged from these findings is the high computational expenses associated with simulation-based optimisation of complex maintenance systems. The ability of simulation to model complex systems comes at the risk of running into high computational expenses. The cost of simulation software and multiple powerful workstations are relatively high. Even then, simulation optimisation will consume a long time as shown in the current research. This is an important issue for future research.

\section{Conclusions and Future Work}

In this paper, the main aim was to explore the optimisation of two maintenance systems from industry. The simulation based optimisation framework for maintenance was applied for the first time on industrial case studies. The research has shown that the framework can guide the process of connecting the simulation model to the optimisation engine in case studies that vary in terms of sector, size, number of manufacturing processes and level of maintenance documentation. Therefore, both academics and practitioners are advised to follow such a comprehensive framework while attempting to optimise maintenance problems.

This study is one of the first to optimise various maintenance strategies simultaneously with their parameters in industrial manufacturing systems while considering production dynamics. Investigated maintenance strategies include $\mathrm{CM}$, PM with PM frequency as a parameter, OM and CBM with both inspection frequency and CBM threshold as parameters. This was possible using the maintenance modelling approach. The results suggest that over-looking the optimisation of maintenance strategies may lead to sub-optimal solutions. The complexity of maintenance problems makes it difficult to assume a given maintenance strategy is the optimum for each asset in the system.

The empirical findings in this study provide a new understanding of nonconflicting objectives in maintenance systems. In some cases, it appears that traditional trade-offs between maintenance cost and production throughput are not present. This is an important issue to consider in future research.

While two specific cases are considered in this research. Together, they cover a range of features that are present in maintenance optimisation problems. The following is a highlight of new insights and contributions to the wider field:

- The two cases demonstrate the application of a generic process for simulation-based optimisation of maintenance systems.

- In an area where industrial case studies are considered rare, the detailed data within these cases presented here can be used as benchmarks in future work applying different methodologies

- This research has shown the significance of optimising the maintenance strategy for each asset in a complex system (for example: CM, PM, OM 
or CBM). This is usually overlooked in the literature where the focus is solely on optimising maintenance parameters assuming a given maintenance strategy is the optimum

- The results have shown that traditional trade-offs between maintenance cost and production throughput may not always be present. Which is an important issue that needs to be taken into consideration when optimising maintenance systems

One of the issues that emerged from these findings is the high computational expenses associated with simulation-based optimisation of complex maintenance systems. Conducting such experiments in timely manner require multiple powerful workstations and inevitably multiple software licenses. A possible area of future research would be to investigate approaches for reducing computational expenses. In addition, the search of the optimisation algorithm in useless space as found in the current research might be reduced to lead to approaches where more efficiency is realised.

\section{Acknowledgements}

The authors would like to thank the University of Jeddah for funding this research.

\section{REFERENCES}

[1] Ding, S. and Kamaruddin, S. (2015), "Maintenance policy optimization: literature review and directions", The International Journal of Advanced Manufacturing Technology, vol. 76, no. 5-8, pp. 1263-1283.

[2] Briš, R. (2008), "Parallel simulation algorithm for maintenance optimization based on directed Acyclic Graph", Reliability Engineering and System Safety, vol. 93, no. 6, pp. 874884.

[3] Gupta, A. and Lawsirirat, C. (2006), "Strategically optimum maintenance of monitoringenabled multi-component systems using continuous-time jump deterioration models", Journal of Quality in Maintenance Engineering, vol. 12, no. 3, pp. 306-329.

[4] Rezg, N., Chelbi, A. and Xie, X. (2005), "Modeling and optimizing a joint inventory control and preventive maintenance strategy for a randomly failing production unit: analytical and simulation approaches", International Journal of Computer Integrated Manufacturing, vol. 18, no. 2-3, pp. 225-235.

[5] Alrabghi, A. and Tiwari, A. (2015), "State of the art in simulation-based optimisation for maintenance systems", Computers and Industrial Engineering, vol. 82, pp. 167-182.

[6] Robinson, S. (2007), Simulation : the practice of model development and use, Wiley, Chichester.

[7] Alrabghi, A. and Tiwari, A. (2016), "A novel approach for modelling complex maintenance systems using Discrete Event Simulation", Reliability Engineering and System Safety, vol. 154, pp. 160-170.

[8] Welch, P. D. (1983), "The statistical analysis of simulation results", in Lavenberg, S. (ed.) Computer Performance Modeling Handbook, Academic Press, New York, pp. 268-328.

[9] Savic, D. A., Bicik, J. and Morley, M. S. (2011), "A DSS generator for multiobjective optimisation of spreadsheet-based models", Environmental Modelling and Software, vol. 26, no. 5, pp. 551-561.

[10] Deb, K. (2011), "Multi-objective Optimisation Using Evolutionary Algorithms: An Introduction", in Wang, L., Ng, A. H. C. and Deb, K. (eds.) Multi-objective Evolutionary Optimisation for Product Design and Manufacturing, Springer, London, pp. 3-34. 
[11] Van Horenbeek, A. and Pintelon, L. (2012), "Optimal prognostic maintenance planning for multi-component systems", European Safety and Reliability Conference: Advances in Safety, Reliability and Risk Management, ESREL 2011, 18-22 September 2011, Troyes, France, Taylor \& Francis Group, London, pp. 910-917.

[12] Johansson, E. C. and Jägstam, M. (2010), "Maintenance planning using simulationbased optimization", Spring Simulation Multiconference 2010, SpringSim'10, 11-15 April 2010, Orlando, Society for Computer Simulation International, San Diego, CA, pp. 1-8.

[13] Alrabghi, A. and Tiwari, A. (2016), "A novel framework for simulation-based optimisation of maintenance systems", International Journal of Simulation Modelling, vol. 15, no. 1.

[14] Hong, D. S., Kamaruddin, S. and Azid, I. A. (2012), "Maintenance policy selection: a review towards building proper selection model", International Journal of Industrial and Systems Engineering, vol. 10, no. 3, pp. 355-375.

[15] Jamshidi, R. and Esfahani, M. M. S. (2015), "Maintenance policy determination for a complex system consisting of series and cold standby system with multiple levels of maintenance action", International Journal of Advanced Manufacturing Technology, vol. 78, no. 5-8, pp. 1337-1346.

[16] Oyarbide-Zubillaga, A., Goti, A. and Sanchez, A. (2008), "Preventive maintenance optimisation of multi-equipment manufacturing systems by combining discrete event simulation and multi-objective evolutionary algorithms", Production Planning and Control, vol. 19 , no. 4 , pp. 342-355.

[17] Deb, K. (2001), Multi-objective optimization using evolutionary algorithms, John Wiley \& Sons, Chichester, England. 
2017-06-20

Simulation-based optimisation of maintenance systems: Industrial case studies

\author{
Alrabghi, Abdullah
}

Elsevier

Abdullah Alrabghi, Ashutosh Tiwari, Mark Savill, Simulation-based optimisation of maintenance systems: Industrial case studies, Journal of Manufacturing Systems, Volume 44, Part 1, July 2017, Pages 191-206

http://dx.doi.org/10.1016/j.jmsy.2017.05.008

Downloaded from Cranfield Library Services E-Repository 